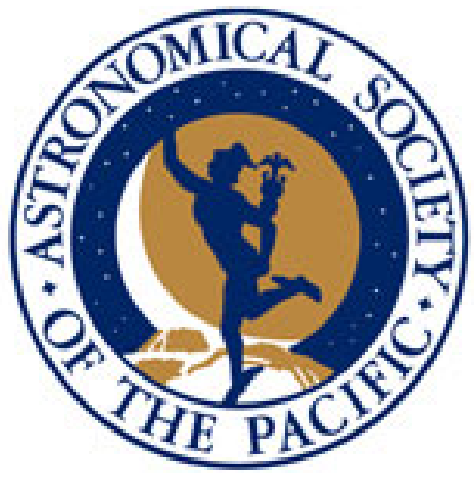

Aperture Photometry Tool

Author(s): Russ R. Laher, Varoujan Gorjian, Luisa M. Rebull, Frank J. Masci, John W. Fowler, George Helou, Shrinivas R. Kulkarni and Nicholas M. Law

Reviewed work(s):

Source: Publications of the Astronomical Society of the Pacific, Vol. 124, No. 917 (July 2012), pp. 737-763

Published by: The University of Chicago Press on behalf of the Astronomical Society of the Pacific

Stable URL: http://www.jstor.org/stable/10.1086/666883

Accessed: 24/09/2012 11:51

Your use of the JSTOR archive indicates your acceptance of the Terms \& Conditions of Use, available at

http://www.jstor.org/page/info/about/policies/terms.jsp

JSTOR is a not-for-profit service that helps scholars, researchers, and students discover, use, and build upon a wide range of content in a trusted digital archive. We use information technology and tools to increase productivity and facilitate new forms of scholarship. For more information about JSTOR, please contact support@jstor.org. 
Publications of the Astronomical Society of the Pacific, 124:737-763, 2012 July

(C) 2012. The Astronomical Society of the Pacific. All rights reserved. Printed in U.S.A.

\title{
Aperture Photometry Tool
}

\author{
Russ R. Laher, ${ }^{1}$ Varoujan Gorjian, ${ }^{2}$ Luisa M. Rebull, ${ }^{3}$ Frank J. Masci, ${ }^{4}$ John W. Fowler,${ }^{4}$ \\ George Helou, ${ }^{4}$ Shrinivas R. Kulkarni, ${ }^{5}$ and Nicholas M. Law ${ }^{6}$ \\ Received 2010 March 29; accepted 2012 May 24; published 2012 July 10
}

\begin{abstract}
Aperture Photometry Tool (APT) is software for astronomers and students interested in manually exploring the photometric qualities of astronomical images. It is a graphical user interface (GUI) designed to allow the image data associated with aperture photometry calculations for point and extended sources to be visualized and, therefore, more effectively analyzed. The finely tuned layout of the GUI, along with judicious use of color-coding and alerting, is intended to give maximal user utility and convenience. Simply mouse-clicking on a source in the displayed image will instantly draw a circular or elliptical aperture and sky annulus around the source and will compute the source intensity and its uncertainty, along with several commonly used measures of the local sky background and its variability. The results are displayed and can be optionally saved to an aperture-photometrytable file and plotted on graphs in various ways using functions available in the software. APT is geared toward processing sources in a small number of images and is not suitable for bulk processing a large number of images, unlike other aperture photometry packages (e.g., SExtractor). However, APT does have a convenient source-list tool that enables calculations for a large number of detections in a given image. The source-list tool can be run either in automatic mode to generate an aperture photometry table quickly or in manual mode to permit inspection and adjustment of the calculation for each individual detection. APT displays a variety of useful graphs with just the push of a button, including image histogram, $x$ and $y$ aperture slices, source scatter plot, sky scatter plot, sky histogram, radial profile, curve of growth, and aperture-photometry-table scatter plots and histograms. APT has many functions for customizing the calculations, including outlier rejection, pixel "picking" and "zapping," and a selection of source and sky models. The radial-profile-interpolation source model, which is accessed via the radial-profile-plot panel, allows recovery of source intensity from pixels with missing data and can be especially beneficial in crowded fields.
\end{abstract}

\section{INTRODUCTION}

Aperture photometry in astronomical image-data analysis is a basic technique for measuring the brightness of an astronomical object, such as a star or galaxy. It is the calculation of source intensity by summing the measured counts from a subimage containing the source (or possibly sources) and subtracting the sky background contribution estimated from a nearby imaged region that excludes the source of interest (Da Costa 1992). The subimage containing the source brightness, or so-called aperture, is a bounding region for the calculation, a

\footnotetext{
${ }^{1}$ Spitzer Science Center, California Institute of Technology, Mail Stop 314-6, Pasadena, CA 91125; laher@ipac.caltech.edu.

${ }^{2}$ Jet Propulsion Laboratory, California Institute of Technology, Mail Stop 169506, Pasadena, CA 91109.

${ }^{3}$ Spitzer Science Center, California Institute of Technology, Mail Stop 220-6, Pasadena, CA 91125.

${ }^{4}$ Infrared Processing and Analysis Center, California Institute of Technology, Mail Stop 100-22, Pasadena, CA 91125.

${ }^{5}$ Caltech Optical Observatories, California Institute of Technology, Mail Stop 249-17, Pasadena, CA 91125.

${ }^{6}$ Dunlap Institute for Astronomy and Astrophysics, University of Toronto, Room 101, Toronto, ON Canada M5S 3H4.
}

two-dimensional area used to define just the portion of a photograph or digital image of the nighttime sky that contains most, if not nearly all, of the observed radiance of the astronomical object under investigation. Conventionally, the aperture is centered on the source of interest, although the calculation is usually insensitive to exact aperture placement, and, in some cases, it is desirable to offset the aperture slightly from the source's center to possibly omit the effect of a neighboring source. The shape of the aperture is circular in its simplest form. Often, the shape of an astronomical object, such as a spiral galaxy viewed at an oblique angle, will determine the aperture shape that is optimal for its scientific study (e.g., elliptical). In addition to geometrical considerations, photometric criteria can govern the aperture's shape (e.g., a set of contiguous pixels in a digital image with data values greater than some threshold). A multiplier greater than one, called an aperture correction, is employed to correct for source intensity outside of the aperture, which is needed for cases where source-crowding effects warrant using a smaller aperture. In theory, an aperture correction is always needed because of limited bandwidth considerations, but, in practice, no aperture correction is made for sufficiently large apertures. As the size of an aperture is increased, the signal from the source 
becomes more fully contained and the noise encompassed by the aperture is increased, and the signal-to-noise ratio $(\mathrm{S} / \mathrm{N})$ of the aperture photometry result is therefore decreased; these considerations mainly influence the size of the aperture chosen for a study.

Aperture photometry calculations, as mentioned above, also normally involve subtracting the contributions to the image data that do not originate from the source of interest, which is generally referred to as the "sky background." An annulus centered on the source may define a region containing the image-data pixels used to locally estimate the background, under the assumption that the background is constant across the aperture. This assumption is violated to varying degrees in the case of crowded fields, depending on the level of the crowding. The annulus is commonly either circular or elliptical, and the annular hole is as large as the aperture or larger, in order to exclude a significant amount of signal from the source of interest for accurate sky background estimation. The inner and outer major and minor radii of an elliptical annulus are the geometrical parameters that determine the number of data samples involved in the background estimation. The outer annular dimensions should be small enough to keep the calculation local to the source, but large enough to contain enough samples to sufficiently minimize the statistical uncertainty.

Aperture photometry, therefore, has its complexities. It is often practical, more instructive, and sometimes more accurate to perform aperture photometry manually on individual sources, rather than to rely on results from automated software programs, such as SExtractor (Bertin \& Arnouts 1996; Holwerda 2005).

The intended audience for this article is anyone who is interested in aperture photometry, including professional and amateur astronomers and astronomy students. We introduce free interactive software called Aperture Photometry Tool (APT) that performs aperture photometry calculations and digitalimage analysis in a highly demonstrative manner. The software is designed to be used with astronomical science images, which are freely available from a variety of public data archives (e.g., the Spitzer Heritage Archive ${ }^{7}$ ). The software is thus suitable for the classroom, but, not only that, it is also an effective analysis tool for astronomical research. This article gives many details about how to use the software and how it works. The objective of the software is to make aperture photometry easy, more accurate, and even fun, through an intuitive graphical user interface (GUI). The software enables aperture photometry to be performed interactively and gives visual feedback in various ways to facilitate learning and calculational refinement. According to Howell (1992), "We are all students in the astronomy game," and, in the context and spirit of that remark, APT was developed to provide a better understanding of aperture photometry and its computed results.

\footnotetext{
${ }^{7}$ See http://sha.ipac.caltech.edu.
}

Our initial goal was to create a GUI-based aperture photometry software application that is instructive on how to perform aperture photometry, but, over time, the fruits of our labor evolved into software that works well enough for professional use in research. APT has been used in the setting of involving teachers and students in original astronomical research as part of the Spitzer Space Telescope Research Program for Teachers and Students (Daou et al. 2005; Rebull et al. 2011) and is now being used in that program's successor, the NASA-IPAC Archive Teacher Research Project (NITARP ${ }^{9}$ ). Moreover, the research has led to new scientific discoveries (Rebull et al. 2011). Generally, APT users report a positive experience with the software, and many find it easy to install on their computers themselves.

The initial beta version of APT was released in 2007 November, and since that time, there have been many releases of the package to add new capabilities and fix bugs. ${ }^{10}$

APT is an object-oriented, all-Java software implementation, and, as such, the same source code is built to generate software packages for Java-capable computers. Currently, four different packages are available to facilitate installation on various types of computers. There are no software dependencies on other astronomical packages or libraries. However, a recently installed version of the Java Runtime Environment (JRE) is required. ${ }^{8}$ Version 2.1.5 of APT is available at the time of completion of this writing, and it was compiled with JDK 1.6.0_31.

The structure of this article is as follows. Section 2 discusses the design considerations that went into creating APT. Section 3 tours the layout of APT's main GUI panel. Section 4 gives basic APT usage instructions for users wanting a quick start. Section 5 explains how APT does sky background estimation and the available options for controlling it. Section 6 provides details on how APT does aperture photometry calculations and what options are available for refining the calculations. Section 7 contains several subsections that discuss APT's salient components, functionality, applicability, and usage: image display, user preferences, output files, columns in the output aperture photometry table, graphs, radial-profile interpolation, pick/zap tool, sourcelist processing, source-list generation, simple photometric calibration, image comparator and blink capability, batch mode, and internationalization. Section 8 covers software limitations and future upgrade plans. A summary is provided in the concluding section.

\footnotetext{
${ }^{9}$ See http://nitarp.ipac.caltech.edu.

${ }^{10}$ APT can be downloaded from http://www.aperturephotometry.org. This Web site has also has information on using APT, including installation instructions for Mac OS X, Linux, Windows, and Solaris machines.

${ }^{8}$ APT requires the following packages: JFreeChart (www.jfree.org), JRegEx (jregex.sourceforge.net), and Jama (math.nist.gov/javanumerics/jama), plus a handful of the Spitzer Science Center Spot/Leopard Java classes for the astrometric calculations. These come packaged with APT, and so the user need not install them separately.
} 
A companion article, in the same issue of the PASP that this article appears, gives a quantitative comparison of calculational results from SExtractor and APT with identical inputs, for the case of noncrowded sources (Laher et al. 2012). The article shows that both software programs give results that are generally in excellent agreement, especially for bright sources, and seeks to find explanations for the discrepancies that occur for faint sources.

Users who want to begin using APT right away may download and install the software, and then skip directly to the tutorial in $\S 4$.

\section{DESIGN CONSIDERATIONS}

APT is meant to complement, rather than supplant, prevailing noninteractive (batch-mode) aperture photometry software programs (e.g., the aforementioned SExtractor ${ }^{11}$ ). APT was modeled after the popular DS9 FITS viewer (Joye and Mandel 2003) in some ways, but with a focus on advanced aperture photometry capabilities. There are other interactive software programs that do aperture photometry, such as IRAF, ${ }^{12}$ which is a well-established workhorse in the astronomical community, but these can be difficult to install and less than straightforward to use, especially for nonspecialists. There are also commercial aperture photometry software packages that are popular with astronomers (e.g., the Interactive Data Language and associated IDL Astronomy User's Library ${ }^{13}$ ), which are available at some cost. APT was designed to address these points and also to have unique features and functions not found in other aperture photometry programs.

Why is it desirable to visualize the data and interact with the aperture photometry calculations? There are many answers to this question, and the problems associated with aperture photometry are not easily realized until one looks at the data. For example, an astronomer may not be aware that an astronomical source of interest is in the "crust" of a mosaicked image, which has a lower depth of coverage and ipso facto implications of higher measurement uncertainty. Or, one may not realize that a source has a significant number of blank or missing pixels (i.e., pixels set to $\mathrm{NaN}$ [not a number] or Inf, [infinity]), even though SExtractor may have yielded FLAGS $=0$ for that source. The accuracy of the background estimation can have a substantial effect on the results, especially for large apertures and relatively faint sources. In many cases, one will want to look at the contents of the image region used for background estimation as a sanity-check and possibly make adjustments.

With the above considerations in mind, the classroomsuitability criteria that we adopted for APT are listed as follows:

\footnotetext{
${ }^{11}$ See http://www.astromatic.net/software/sextractor.

${ }^{12}$ IRAF stands for Image Reduction and Analysis Facility; see http://iraf.noao .edu and Tody $(1986,1993)$.

${ }^{13}$ See http://idlastro.gsfc.nasa.gov.
}

visualization of inputs and outputs, user interaction, ease of use, ability to run on a variety of machines and operating systems, ease of installation, and zero acquisition cost.

Data visualization pertains to graphical and statistical representations of not only the aperture photometry results, but also the input data. We found that showing an overlay of the aperture and sky annulus on the input image was important in giving the user important visual information for setting up the aperture photometry calculation in relation to the astronomical source of interest and its environs. APT's GUI has many controls that promote user interaction, such as changing the size of the aperture and sky annulus, and then immediately seeing the resulting overlay. The feedback provided by APT's various graphs allows the user to make intelligent choices in modifying the input parameters. The overall effect of equipping APT with a rich palette of controls and capabilities is to engage the user, so that the user will want to spend time running the software. At least, this is our aim.

It is important to us that the software is able to be executed on a variety of machines, particularly Mac, Windows, and Linux computers, which are the most popular today. It is for this reason that we chose to implement APT in the Java programming language. In fact, any computing platform that runs the Java Virtual Machine (JVM) can run APT. We also programmed APT with special functionality to enable it to run on computers with smaller memories and with smaller screens. APT's minimum memory requirement is, by design, very modest, only around 300 Mbytes with a $2048 \times 4096$-pixel image loaded. This is small enough to accommodate older machines often found in the classroom. APT can be easily used on machines with relatively small memories to analyze portions of very large images. This is effected by configuring APT's maximum image size to as little as 500 pixels on a side. APT can also be set up with a compact-sized GUI that fits on some computer screens that are smaller than normal in size, such as those on the smaller laptops.

APT has relatively simple installation instructions (some APT users have reported that APT is much easier to install than IRAF). We have eliminated all high-level software dependencies by putting it in a single package to simplify the process. Additionally, we have refined the installation process down to just a handful of steps. APT is especially easy to install on a Mac.

Finally, the software can be downloaded via the Internet and used free of charge for research and education in astronomy and astrophysics, satisfying the last criterion in our list of classroomsuitability attributes.

\section{MAIN GUI PANEL}

This section summarizes the prominent features of APT's main GUI panel, with more details given later in $\$ 7$. The layout of APT's main GUI panel is shown in Figure 1, and the enumeration below in this section refers to the numbered items in the figure. The computer screen shots in Figure 1 and other 


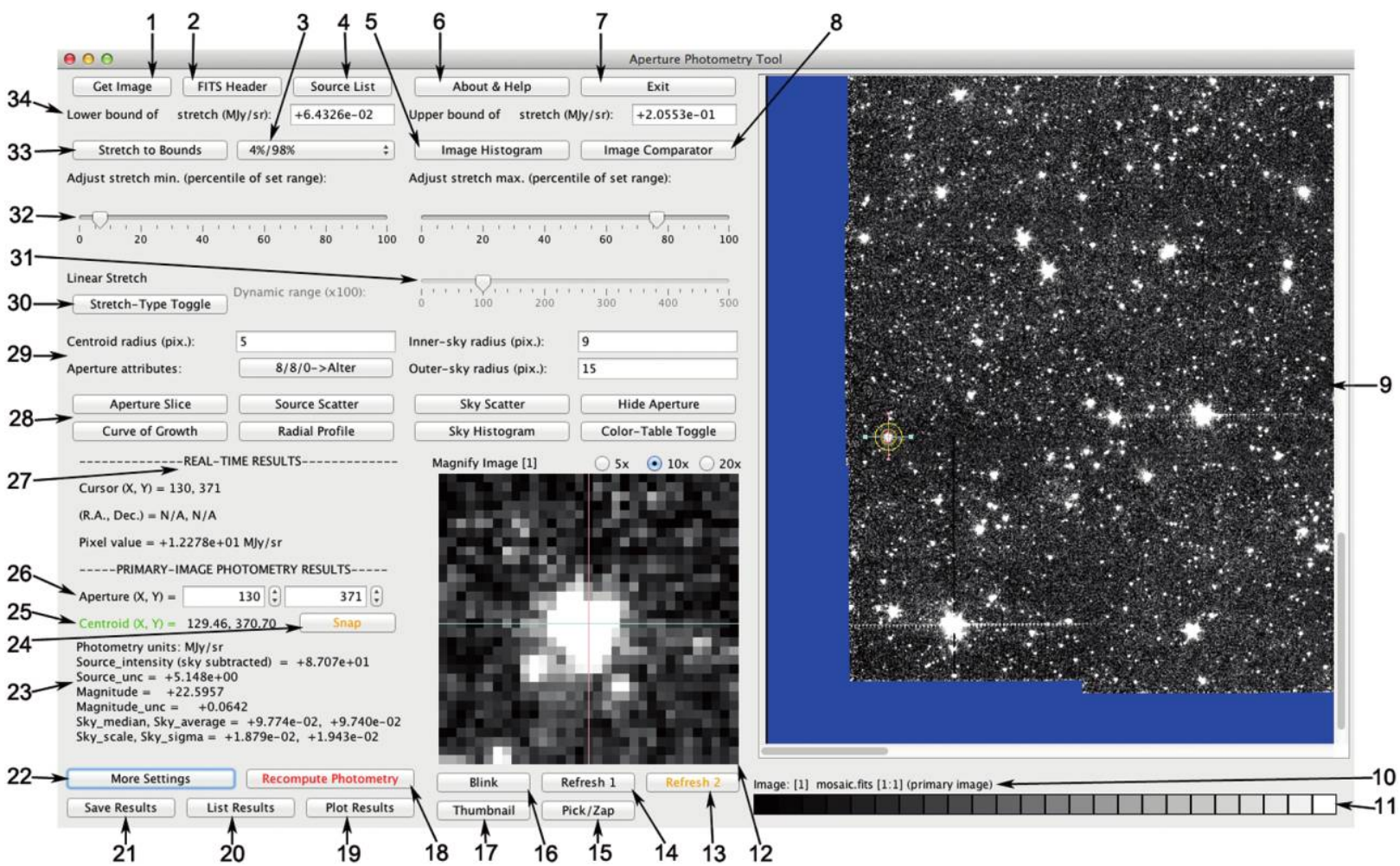

FIG. 1.-APT's main GUI panel. The numbers with arrows highlight the components that are described in $\S 3$.

figures shown in this article were taken on an Apple computer running OS X Lion and will have slightly different appearances under other operating systems. When running the software, hovering the mouse cursor over a widget in the GUI will cause a short pop-up explanatory note or tool tip to be displayed.

Not shown in Figure 1 are the Preferences, File, and Tools pull-down menus, which are located at the top of the computer screen in the case of Macs, or in the main GUI panel's upper-left corner in the case of non-Mac machines. Section 7.2 gives more information about the Preferences menu. Currently, the File menu only has one option, which is to clear the contents of the output aperture-photometry-table file. Section 7.10 discusses the tool-menu option of performing simple photometric calibration of astronomical sources extracted from an image.

The remainder of this section gives descriptions for the numbered items called out in Figure 1:

1. The Get Image button is used to load an image and display it in the GUI. The actions associated with this button have functionality to load either a primary image or a comparator image. The primary image is, by definition, the first image loaded, and the subsequently loaded images are called comparator images, whose purpose is visual comparison with the primary image (see $\S 7.11$ ). Up to three comparator images are permitted. Aperture photometry calculations are done only for the primary image. APT can read only FITS-formatted images. ${ }^{14}$ This includes single-extension FITS files, FITS files with multiple image-data planes in a single image extension, and FITS files with multiple image extensions (but not those with binary-table extensions).

2. The FITS header button pops up a panel that lists the FITS header of the primary image. In the case of a multiextension FITS file, the headers of all extensions are listed. The top of the panel has functionality for case-sensitive searches, which is useful for finding particular keywords, values, and comments in the FITS-header listing.

3. The pull-down menu with the default label $1 \% / 99 \%$ has several options for setting the limits of the image-display stretch, which is the mapping of pixel intensities to values in the 0-255 range for 8-bit graphics. The options include the image-data minimum and maximum, as well as various combinations of image-data percentiles. As the default label indicates, the default setting for stretch is the selection with the 1 and 99 percentiles. Located above this pull-down menu on the main GUI panel are text fields that display the lower and upper bounds of the stretch corresponding to the menu selection made (see item 34 below).

\footnotetext{
${ }^{14}$ FITS stands for Flexible Image Transport System; see http://fits.gsfc.nasa gov and Wells et al. (1981).
} 
4. The Source List button pops up a panel for performing a large number of aperture photometry calculations at source positions that are read from a source list (see § 7.8). From this panel, additional functionality for generating a source list automatically is available (see $\S 7.9$ ).

5. The Image Histogram button pops up a panel with a histogram plot for the entire primary image (see Fig. 2). The lower and upper limits of the graph's horizontal axis are set by the image-display stretch bounds. This histogram is, of course, useful for determining the best stretch. There is an inset table of global-image-data statistics on the graph: minimum, maximum, mean, sigma (standard deviation), median, scale (a robust measure of the data dispersion, given by one-half the difference between the 84.1 and 15.9 percentiles, which is equivalent to 1 standard deviation in the case of Gaussian-distributed data), 1 and 99 percentiles, number of data values used in computing the statistics, and number of pixels set to either $\mathrm{NaN}$ or Inf in the image data. The intent of the displayed statistics is to make the user aware of the image-data contents. Except for the number counts, the data units of the image statistics are given by the image's BUNIT FITS-header keyword, or, if BUNIT is not available, the default is D.N. (digital or data number). The default setting can be changed via the Set Default Image-Data Units option in the Preferences menu.

6. The About \& Help button pops up a panel showing the software version number and the built-in documentation.

7. The Exit button terminates the software program in a user-friendly way (one more chance is given to cancel the termination).

8. The Image Comparator button pops up a panel displaying the primary image on the left side of the panel and up to three comparator images on the right for a side-by-side comparison (see $\S 7.11)$.

9. The main image-viewing panel displays either the primary image or a comparator image (see item 16 below). If the image is bigger than the viewing window, then the scroll bars on the window can be used to view the desired image portion (the user may have to mouse-click on the image to make the scroll bars appear). Bounding-box graphics are overlaid onto the image just outside the visible portion of the image, in order to visually define the region updated by the Refresh 1 button (see item 14 below). The example image in Figure 1 is a Spitzer Space Telescope mosaic, with pixels having missing data represented by the blank color blue. The left-middle side of the image containing data has an aperture and sky annulus overlay, which is drawn by the software when the user mouse-clicks on a source in the displayed image.

10. The filename label indicates the image currently displayed in the main image-viewing panel (see previous item). The label is embedded with additional useful information. The number enclosed in the first set of square brackets indicates whether the currently displayed image is the primary image (value is 1) or a comparator image (value is greater than 1). The two numbers separated by a colon and enclosed within the second set of square brackets indicate the image-extension number and the image-data-plane number within the image extension, respectively.

11. The image-display graphic shows the currently selected display type and color table. The available types are grayscale, inverted grayscale, false color, and inverted false color. There are two false-color tables available: one is called contour because it seeks to differentiate adjacent colors in the color table,

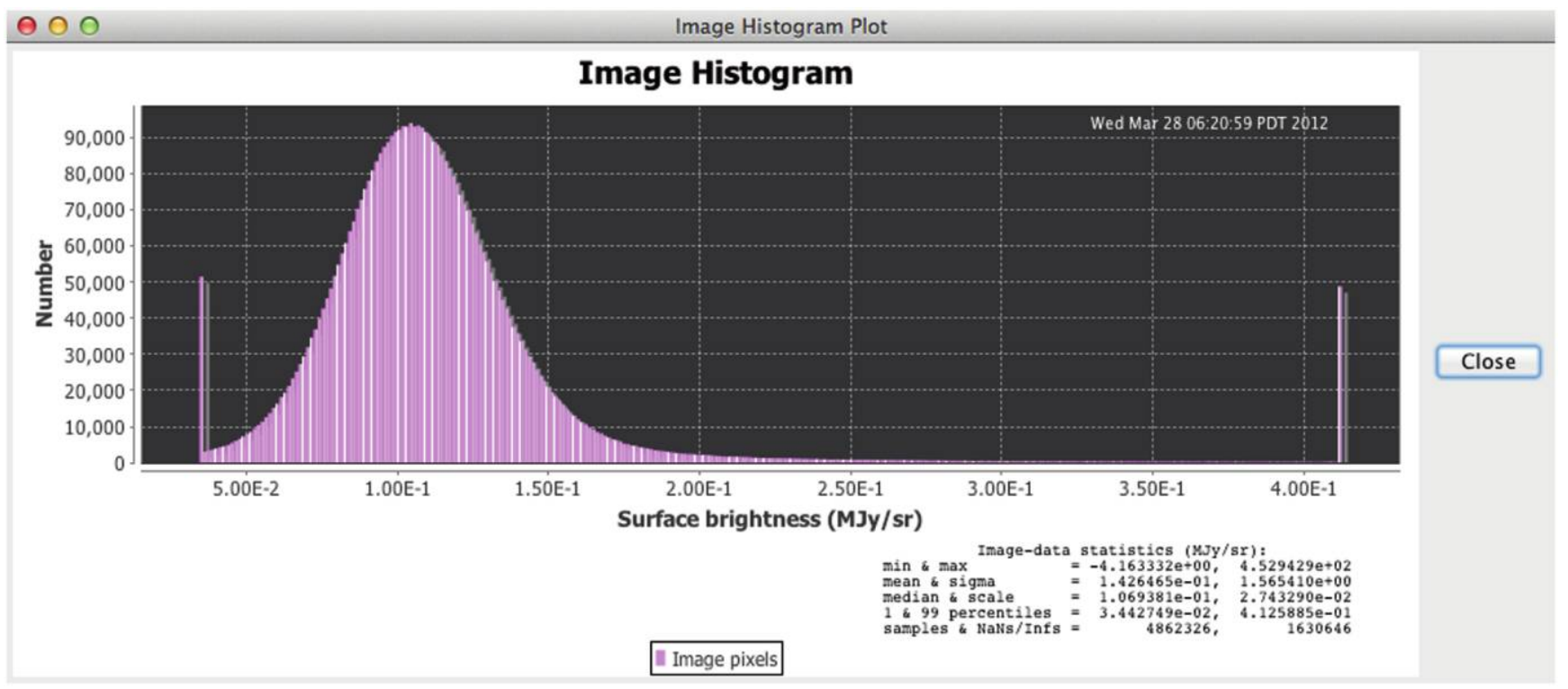

FIG. 2.-Sample image-histogram plot generated by APT. 
and the other (eponymous) color table has a gradation of rainbow colors. There are 24 levels of grayscale or 24 hues in the color tables. The display type and color table can be set via options in the Preferences menu. See item 28 below for related information on the Color-Table Toggle button.

12. The image-magnifier panel displays a subimage of the image currently shown in the main image-viewing panel. The subimage is centered at the mouse-cursor position in the main image-viewing panel. The subimage is changed in real time as the mouse cursor is moved. The magnification options of the imagemagnifier panel are $5 \times, 10 \times$ (the default setting), and $20 \times$.

13. The Refresh 2 button repaints the image for both visible and nonvisible portions of the image in the main image-viewing panel. This is useful after image-display characteristics, such as the stretch or color table, have been changed. After the short amount of time required for this operation to complete, the main image-viewing panel's scroll bars can be used to quickly scroll about the entire image, which will be thereafter displayed with the same image-viewing characteristics (until such time that image-viewing changes are made again).

14. The Refresh 1 button repaints the visible portion of the image in the main image-viewing panel, plus the relatively small nonvisible portion that lies within the bounding-box graphics (see item 9). This option is faster than the repainting of the entire image done by the Refresh 2 button and is mainly useful for redrawing the bounding-box graphics and eliminating any residual bounding-box graphics in the visible portion of the displayed image. The latter can occur after the scroll-bar positions of the main image-viewing panel have been moved or after the main GUI panel has been enlarged. For the most part, the visible portion of the image is repainted automatically after changes to image-viewing characteristics, such as the stretch or color table.

15. The Pick/Zap button pops up a panel with the pick/zap tool (see \$7.7).

16. The Blink button is for image blinking the primary image and up to three comparator images (see $\S 7.11$ ).

17. The Thumbnail button pops up a panel that is capable of displaying the entire primary image, rather than just the portion of it that may be currently displayed in the main image-viewing panel. At the top of the thumbnail panel are a Show Grid button and a display of real-time mouse-cursor position in both image and equatorial coordinates, as well as a display of the real-time pixel-data value at the cursor position. The Show Grid button will overlay a grid labeled with equatorial coordinates and then subsequently transform into a Hide Grid button. If a world coordinate system (WCS) is not available in the image's FITS header, then the grid overlay will be disabled. The thumbnail image can be made to fit on the user's computer screen using the Set Maximum Thumbnail Size option in the Preferences menu. If it is set to larger than the user's screen, then the panel will be automatically scaled to fit and scroll bars will appear. Figure 3 shows an example primary-image thumbnail with a coordinates-grid overlay.
18. The Recompute Photometry button repeats the aperture photometry calculation after changes to its setup have been made, such as different aperture geometrical parameters. Such changes, which affect the results, cause the Recompute Photometry button text to change from the color black to the color red as a reminder to the user that the calculation needs to be updated. More details about how this button works and is used are given in $\S \S 4,6,7.7$, and 7.8.

19. The Plot Results button pops up a panel that allows the setting up of scatter plots of one data column in the output aperture photometry table versus another. Histograms of data columns can also be plotted. Section 7.5 describes the available functionality in more detail.

20. The List Results button pops up a spreadsheet-style listing of the output aperture photometry table. The data columns in the table are fully described in $\S 7.4$.

21. The Save Results button stores a record of the latest aperture photometry calculation as a single row in the output aperture-photometry-table file. A calculation may be manually repeated many times with different settings, but it is not saved in the file until this button is utilized, and then only the last result is saved.

22. The More Settings button pops up panel that enables parametric changes to the aperture photometry calculation, including the specification of source and sky models. The options and controls on this panel are fundamental to utilizing APT to its fullest and are described in $\S \S 4,5$, and 6 , as well as mentioned throughout the remainder of this article.

23 . The main results of the latest aperture photometry calculation are displayed near the lower-left corner of the main GUI panel, under the heading PRIMARY-IMAGE PHOTOMETRY RESULTS. Section 7.4 defines the displayed quantities, which are among the quantities written to the output aperture photometry table when the user mouse-clicks on the Save Results button.

24 . The Snap button nudges the aperture onto the computed centroid of the source of interest (see $\S 6$ for more details, including the color-coding).

25 . The source's centroid position, in floating-point pixels, is displayed just to the left of the Snap button (see $\S 6$ ).

26. The aperture's position, in integer pixels, is displayed in spinner-controllable text fields and can be changed here via either text-field editing or mouse-clicking on the tiny increment/ decrement buttons located just to the right of the corresponding text field.

27. The real-time pixel coordinates, the corresponding equatorial coordinates, and the image-data value at the position of the mouse cursor in the main image-viewing window are displayed under the heading REAL-TIME RESULTS. The representation of the sky coordinates can be changed to either sexagesimal or decimal degrees via theSet Celestial-Coordinates Units option in the Preferences menu. The image-data units are given alongside the image-data value. The default image-data units are used 
000

Primary-Image Thumbnail

\begin{tabular}{l|l|} 
Cursor $(X, Y)=1483,4087$ & Pixel value $=$ \\
(R.A., Dec. $)=104.9727,+16.8501$ degrees & \\
\hline
\end{tabular}

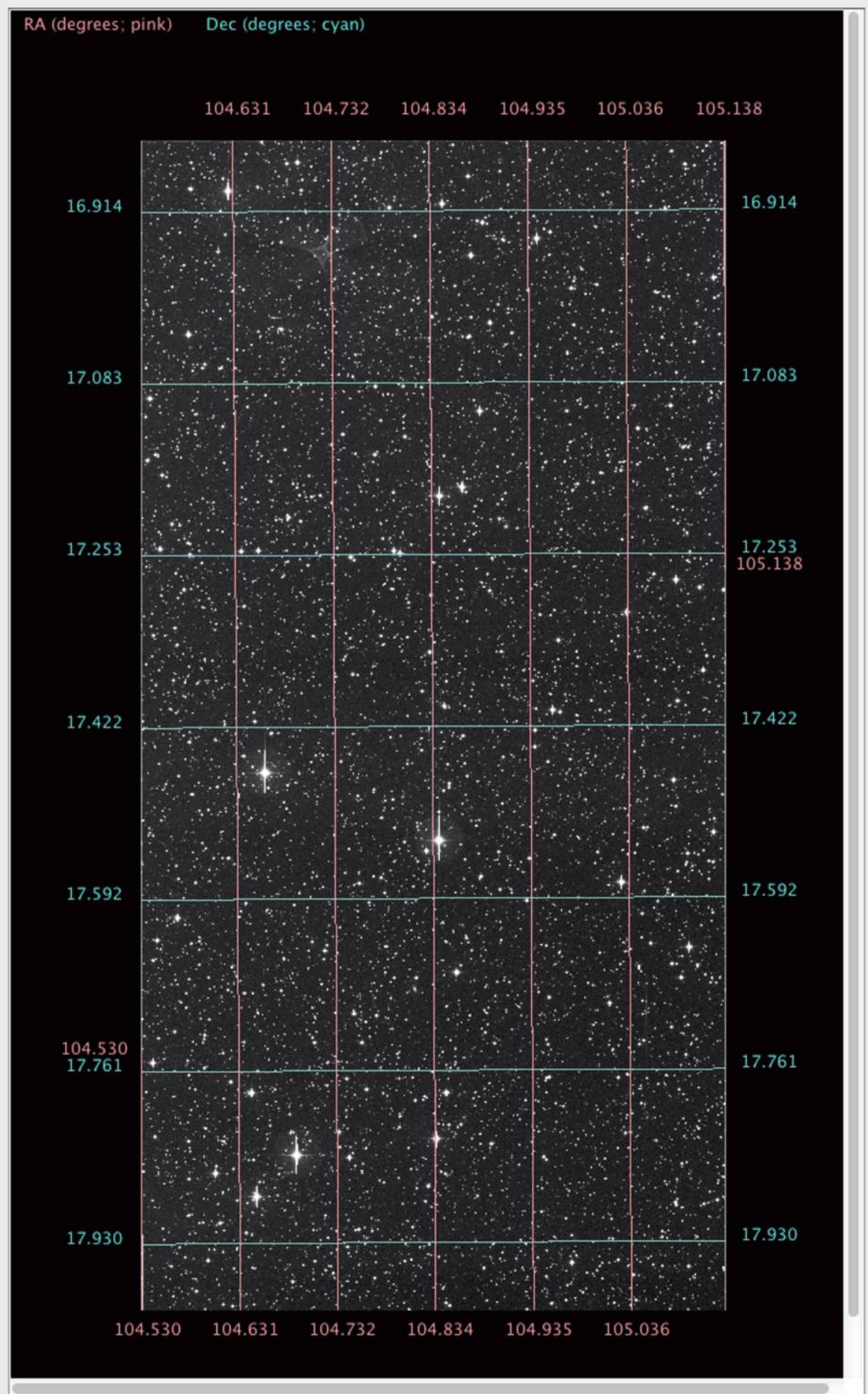

FIG. 3.-APT's primary-image thumbnail. 
when the BUNIT keyword is absent from the image's FITS header and can be set via the Set Default Image-Data Units option in the Preferences menu.

28. The six buttons on the left of the button group, Aperture Slice, Curve of Growth, Source Scatter, Radial Profile, Sky Scatter, and Sky Histogram, pop up various graphs associated with the current calculation (see $\S 7.5$ ). On the right, the Hide Aperture button temporarily hides the aperture overlay and subsequently transforms into the Show Aperture button and the Color-Table Toggle button that successively switches to the available presequenced color-table options (see item 11 above).

29. The Alter button next to the aperture-attributes label pops up a panel that allows changes to the elliptical aperture's major and minor radii and rotation angle (see Fig. 4), and the button's label indicates the values of these parameters. The text fields in the group allow changes to values of the centroid, inner-sky, and outer-sky major radii, in integer pixels. The default aperture shape is circular, in fact, and the default settings for the centroid, aperture, inner-sky, and outer-sky radii are 5, 5, 8, and 15 pixels, respectively. These defaults can be changed via the Preferences menu.

30. The Stretch-Type Toggle button cycles the image-display stretch from linear to logarithmic to histogram equalization and then back to linear.

31. The dynamic range slide control allows the dynamic range of the logarithmic image-display stretch to vary from 0

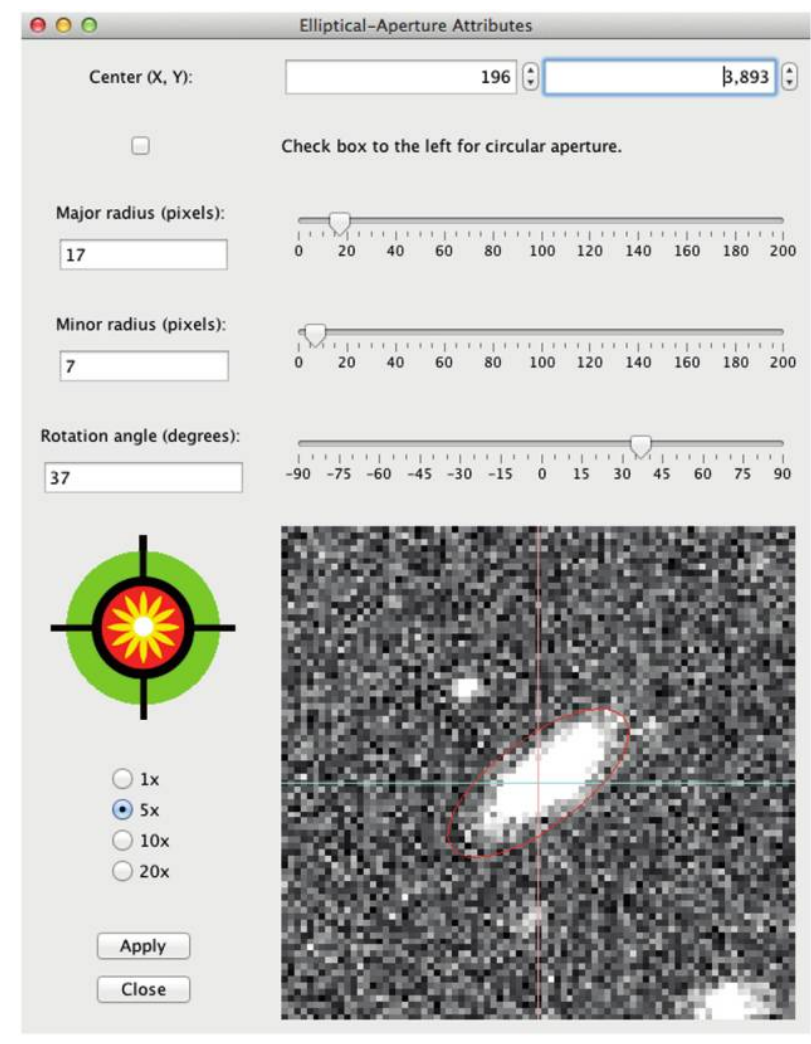

FIG. 4.-APT's panel for setting the elliptical-aperture attributes. (equivalent to a linear stretch) to 5 orders of magnitude (which visually differentiates the smallest image-data values that are above the lower bound of the stretch). The default setting is one order of magnitude. The slide control is disabled for nonlogarithmic types of stretches.

32. The stretch minimum and stretch maximum slide controls allow the lower and upper limits of the image-display stretch, respectively, to be varied from their current settings. The new stretch is instantiated only after the Stretch to Bounds button is pressed (see next item).

33. The Stretch to Bounds button sets the limits of the imagedisplay stretch to the two values in the lower-bound and upperbound text fields (see next item).

34. The lower and upper limits of the current image-display stretch can be manually changed by typing new values into the lower-bound and upper-bound text fields, respectively. The user must either press Enter on the keyboard or mouse-click on the Stretch to Bounds to apply changes made directly to these text fields.

\section{BASIC USAGE INSTRUCTIONS}

APT is intended to be simple to use. Basically, one displays a FITS image and then mouse-clicks on a source (i.e., an astronomical object) shown in the main image-viewing panel to overlay an elliptical aperture onto it. The latter action causes the software to automatically perform an aperture photometry calculation. The computed quantities include, among others, source centroid position, source intensity, source-intensity uncertainty, sky background level, and sky background dispersion width. The default sky algorithm is no sky background subtraction from the source intensity, and the reason for this is to facilitate proper use of APT's radial-profile interpolation capability. More often than not, however, the user will require the sky background to be subtracted from the source intensity, in which case this sky model can be selected from the control panel that pops up after mouse-clicking on the More Settings button (located in the lower-left corner of the main GUI panel; see also item 22 in $\S 3$ ). See Figure 5 for a depiction of the More Settings panel.

The general flow of the work progresses from the buttons and controls in the upper-left region of the main GUI panel to the middle-left region and then lower-left region of the same. Here are the basic instructions.

1. Take a moment to review the default settings by selecting List Preferences from the Preferences menu. More information on user preferences and how to change them is given in $\S 7.2$.

2. Choose a primary image to display by mouse-clicking on the Get Image button in the upper-left corner of the GUI panel. The primary image, as defined here, is the first image displayed in the main image-viewing panel (after the primary image is loaded, a subsequent mouse-click on the Get Image button will 


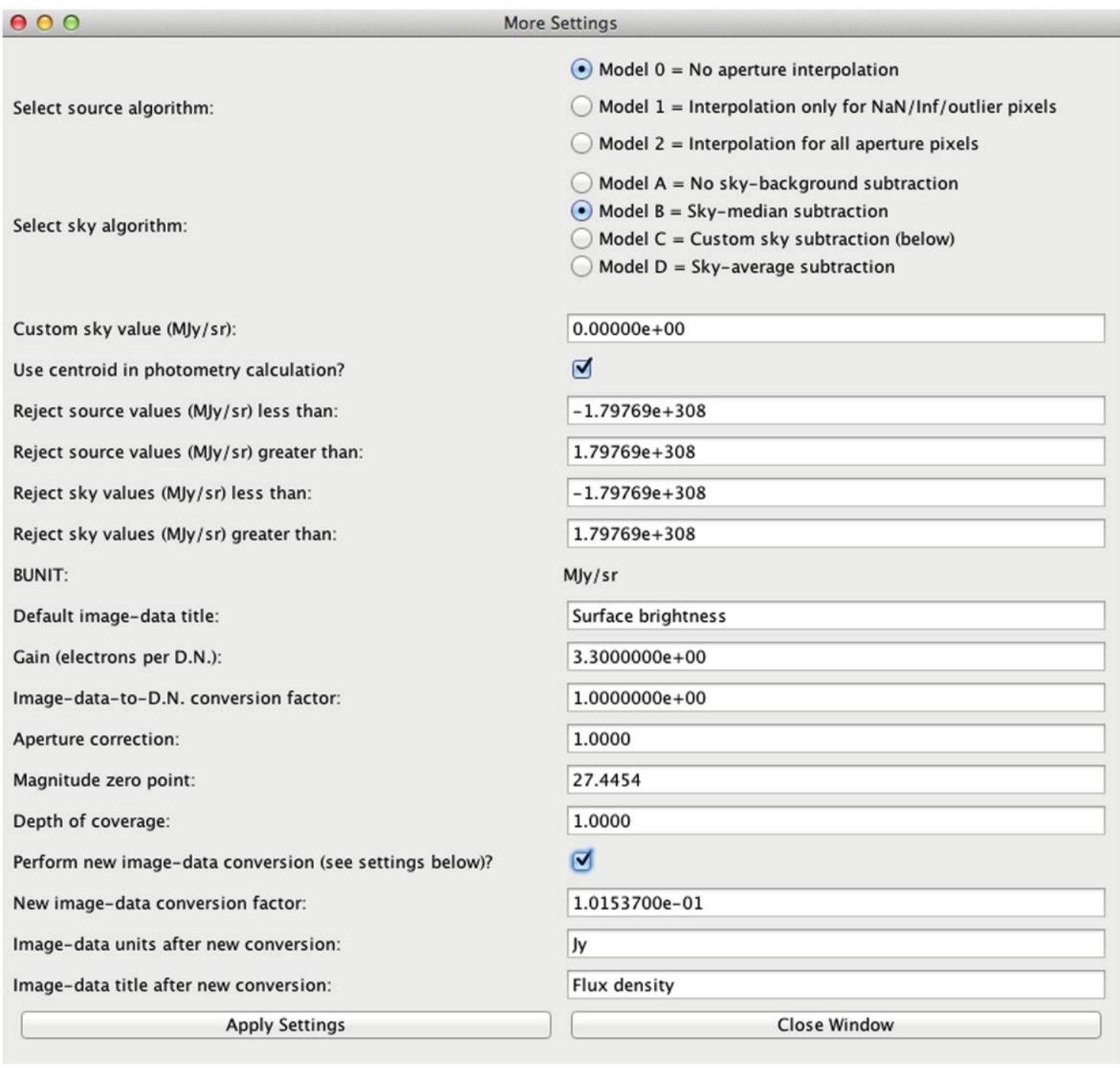

FIG. 5.-APT's More Settings panel.

allow the user to load either a different primary image or comparator images).

3. Adjust the image-display stretch for best viewing. As an aid, click on the Image Histogram button to see the stretch range spanned by the image.

4. Select centroid and sky annulus major radii (integer values only), and click on the Alter button beside the aperture-attributes label to select the elliptical aperture attributes, as appropriate for the source of interest.

5. Place the mouse cursor over the source of interest in the image displayed in the main image-viewing panel and click to overlay an aperture.

6. Show and study the various graphs (instructions are given in $\S 7.5)$.

7. Select the desired new radii, as appropriate, and/or change other settings as needed.
8. Redraw or overlay a new aperture by either clicking on the Recompute Photometry button or clicking on the Snap button for nudging the aperture onto the source centroid location or placing the mouse cursor on the image and clicking.

9. If necessary, increment or decrement the spinner controls for fine-tuning the aperture's position.

10. Click on the Recompute Photometry button to redraw/ overlay a new aperture.

11. Show and study the various graphs again.

12. Optionally click on the Save Results button, located in the lower-left corner of the main GUI panel, in order to save/ append the results to APT's output photometry-table file (e.g., APT.tbl). The adjacent List Results and Plot Results buttons can be used to list and plot the saved results.

13. Repeat the above steps for each source of interest. 


\section{SKY BACKGROUND ESTIMATION}

Strictly speaking, the background should be the best estimate of the true underlying background emission, excluding contamination from the source flux being measured, and not biased by any other neighboring sources or outliers. As a practical matter, we estimate the background in the aperture from an elliptical sky annulus surrounding it, and this method does not account for gradients in the sky background caused by sources in the annulus. A bright source in the sky annulus contributes to the background in the aperture, and its effect is not necessarily something that is to be completely ignored or filtered out, which is why APT has a variety of sky models from which to choose.

APT has fairly straightforward methods for estimating the sky background in the region local to the source of interest, and there are a few options available for controlling how it is done. Only image pixels in an elliptical sky annulus centered on a user-selected center position are considered for the background calculation. The pixels with $\mathrm{NaN}$ or Inf are rejected outright. The center position, for purposes of background estimation, is specified in integer pixels only. There are three different ways of specifying the center position in APT:

1. Mouse-clicking on the image displayed in the main imageviewing panel.

2. Changing the values in the spinner-controllable text fields for the aperture position, which are located near the lower-left corner of the main GUI panel.

3. Clicking on the Snap button in the lower-left corner of the main GUI panel (more on this in $\S 6$ ).

The inner and outer major radii of the sky annulus, in integer pixels only, can be specified on the main GUI panel. These major radii are used to scale the ellipse specified for the aperture. The inner radius of the sky annulus must be greater than or equal to the radius of the elliptical aperture along any direction from the center of the ellipse. No limitation is placed on the outer radius of the sky annulus, except that it must be greater than the inner radius. The default values that specify the size and shape of the aperture and sky annulus, which are loaded when APT is launched, can be specified via the Set Photometry Size Parameters option under the Preferences menu.

On the APT control panel that pops up after clicking on the More Settings button, the user can select from one of four available sky models:

Model A.-No sky background subtraction.

Model B.-Sky median subtraction.

Model C.-Custom sky subtraction.

Model D.-Sky average subtraction.

Sky median subtraction is less sensitive than sky average subtraction to other bright sources that may fall within the sky annulus, which might otherwise cause the background to be overestimated. If model $\mathrm{C}$ is specified, then the custom value to be used must be specified in the text field labeled Custom sky value on the More Settings panel.
The More Settings panel has text fields where the user can optionally specify lower and upper thresholds for the rejection of outlier pixels in the sky annulus from the background calculation. The default values for the lower and upper thresholds are the largest possible negative and positive double-precision numbers, respectively, so that, by default, no pixels are rejected. The values for the outlier-rejection thresholds must be given in the data units of the image's FITS file. It is best to study the various aperture photometry graphs provided by APT, in order to figure out the best thresholds, and then set these thresholds before optionally converting the image-data units into the desired source-intensity units (see $\S 7.5$ for how to do the latter).

In addition to the aforementioned outlier rejection, there is yet another outlier-rejection method that is applied. The median and standard deviation are computed, and all data values that lie greater than 3 standard deviations from the median are rejected. Currently, this number of standard deviations is hard-coded and cannot be changed by the user.

The pixel zap functionality of the pick/zap tool can also be used to temporarily eliminate pixels from the background calculation. More details about the pick/zap tool are given in $\S 7.7$.

The median and average of the remaining image data in the sky annulus after the outlier rejection have been applied are computed as possible background estimators. The median or average times the number of pixels in the aperture form a product that is the sky contribution optionally subtracted from the integrated image data of the source to get the backgroundsubtracted source intensity. These quantities are labeled Sky_ median/pix and Sky_average/pix, respectively, in the output aperture photometry table (see $\S 7.4)$. The standard deviation is computed for the Sky_sigma column in the aperture photometry table. Likewise, the root-mean-squared (rms) value is computed for the Sky_RMS/pix column. The Sky_scale column, a robust estimator of the data dispersion, is computed as one-half of the difference between the 84 and 16 data percentiles.

\section{APERTURE PHOTOMETRY IMPLEMENTATION}

The aperture photometry calculation primarily yields the source intensity and its uncertainty. The former involves summing pixel values within the aperture to get the total intensity, then subtracting the product of the aperture area, in pixels ${ }^{2}$, and the per-pixel sky background, in order to get the source intensity. The latter also requires the aperture and sky annulus geometry, as well as extra information, including the detector gain, the conversion factor from image-data units to D.N. (if image is not already in units of D.N.), the background-estimation method, and the sky background standard deviation. APT works under the assumption that the background is constant across the aperture.

APT performs its calculations with an elliptical aperture, which the user specifies with major and minor radii and a rotation angle. These quantities are recorded in the Maj $R \_a p e r$, MinR_aper, and Rot_aper columns, respectively, in the output 
aperture photometry table (see $\S 7.4$ ). Of course, APT also allows circular apertures, as a circle is a special case of an ellipse with its major radius equal to its minor radius.

The basic inputs for the calculation are the elliptical-aperture geometrical parameters, the source centroid major radius, and position coordinates of the aperture center (the instructions for selecting these quantities are given in $\S 5$ ). The calculation of the source's centroid position, which shares the same center position as the aperture, can involve a different number of pixels than used in the calculation. The source centroid ellipse is its major radius scaled to the ellipse specified for the aperture. The aperture geometrical parameters and centroid major radius can be specified on the main GUI panel.

The More Settings panel has text fields where the user can optionally specify lower and upper thresholds for rejection of spurious aperture pixels in the calculation. The default values for the lower and upper thresholds are the largest possible negative and positive double-precision numbers, respectively, so that, by default, no pixels are rejected. Again, values for outlier-rejection thresholds must be given in image-data units.

The pixel zap functionality of the pick/zap tool can also be used to temporarily eliminate pixels from the calculation. More details about the pick/zap tool are given in $\S 7.7$.

The method that computes the source centroid position is iterative and runs for 100 iterations. The first iteration is bootstrapped from the user-selected aperture position. The $k+1$ th iteration computes the following $x$ and $y$ image coordinates of the source centroid:

$$
x_{\text {centroid }}^{k+1}=x_{\text {centroid }}^{k}+\frac{\sum_{i, j \in S(k)}\left(x_{i}-x_{\text {centroid }}^{k}\right)\left(d_{i j}-d_{\text {min }}^{k}\right)}{\sum_{i, j \in S(k)}\left(d_{i j}-d_{\text {min }}^{k}\right)},
$$

and

$$
y_{\text {centroid }}^{k+1}=y_{\text {centroid }}^{k}+\frac{\sum_{i, j \in S(k)}\left(y_{j}-y_{\text {centroid }}^{k}\right)\left(d_{i j}-d_{\text {min }}^{k}\right)}{\sum_{i, j \in S(k)}\left(d_{i j}-d_{\text {min }}^{k}\right)},
$$

where the sums are over pixels in the centroid ellipse $S(k)$ that meet criteria given below; image data value $d_{i j}$ is located at pixel $\left(x_{i}, y_{j}\right)$; and $d_{\min }^{k}$ is the smallest data value in the centroid ellipse. The data values included in the summing must be greater than $d_{\min }^{k}$, not NaN or Inf, and less than or equal to the upper outlier-rejection threshold for the calculation. The centroid ellipse is allowed to move with each iteration, so it is necessary to recompute $d_{\min }^{k}$ each time. The centroid calculation is done with subpixel resolution, but the step size is currently limited to no less than 0.05 pixels for computational speed. The method generally converges for isolated sources, but not always, and the user is cautioned to check that the resulting source centroid is a reasonable one. The source centroid calculation does not always give the best aperture position for the source of interest, especially if there are other sources nearby that fall within the centroid ellipse. One can use visual feedback from the aperture-slice and source-scatter graphs for improved manual aperture positioning. See $\S 7.5$ for more information about the available APT graphs.

The More Settings panel has radio buttons for the user to select one of three available source models:

Model 0.-No aperture interpolation.

Model 1.-Aperture interpolation only for $\mathrm{NaN}$ and Inf pixels (including zapped pixels).

Model 2.- Interpolation for all aperture pixels.

Model 0 will underestimate the source intensity if there are a significant number of blank pixels in the aperture. Model 1 was designed to remedy this, but it requires that the user set up a radial-profile model for the interpolation, and APT has a tool that makes it easy (as discussed in $\S 7.6$ ). Model 2 uses the radial-profile model to compute data values for all pixels in the aperture and generally gives a result that is within a few percent or better of model 0 if the radial-profile model was set up on the same source. Model 2 is most useful in cases where the source of interest has missing aperture pixels and the radial-profile model was set up on a different source that has no missing pixels, which is facilitated by the built-in automatic scaling and offsetting of the radial profile.

The aperture photometry calculation is done with subpixel resolution. The default subpixel size is 0.01 pixels. The small value can cause the computations to take several seconds for very large aperture ellipses. The subpixel size can be changed via the Set Calculation Step Size option in the Preferences menu.

By default, the calculation is performed with the aperture centered on the calculated source centroid. The Centroid $(X, Y)=$ label on the lower-left side of the main GUI panel is displayed in the color green to indicate that centroiding is enabled and in the color black to indicate that it is disabled. Unchecking the Use centroid in photometry calculation? check box on the More Settings panel will do the disabling and cause the calculation to revert to centering the aperture on the integer pixel coordinates of the selected aperture position. Note that although centroiding moves the center position of the aperture with subpixel resolution, the center position of the sky annulus is incremented only with integer-pixel resolution.

The Snap button in the lower-left main GUI panel is available to nudge the aperture onto the computed source centroid location. After moving the aperture, it automatically recomputes the photometric results (just like the Recompute Photometry button). If centroiding is turned on and the aperture is already fairly close to the centroid, the recomputation may give the same nonbackground-subtracted source intensity as before (with slight changes possible to the source-intensity uncertainty, sky background, and sky background dispersion), and the main difference will be that the aperture will appear to be better centered on the centroid position (and the data points plotted 
in most of the APT graphs will be shifted accordingly). If centroiding is turned off, the user will obtain a new result at the new aperture position, which, after snapping the aperture, will be the centroid position represented by integer image coordinates. The Snap button text will turn the color yellow to remind the user to mouse-click on this button to fully center the aperture on the centroid location. This color reminder can and should be ignored if the user wants photometric results for an aperture that is dislocated from the centroid position, in which case centroiding should be turned off.

The source-intensity-uncertainty calculation requires the detector gain (electrons per D.N.), the conversion factor from image-data units to D.N., and the depth of coverage. The default value for these quantities is 1.0. When a primary image is loaded, the software attempts to read the GAIN FITS keyword and, if found, automatically overrides the default gain value. For image data that are already in units of D.N., the value of 1.0 is appropriate for the image-data-units-to-D.N. conversion factor. A depth of coverage of 1.0 is correct for a single observation. These quantities can be subsequently overridden on the More Settings panel, after an image has been loaded. The default gain value, used in the absence of the GAIN FITS keyword, can be changed via the Set Default Image-Data Gain option in the Preferences menu.

APT computes the source-intensity uncertainty, $\sigma_{\text {src }}$, using

$$
\sigma_{\text {src }}=\sqrt{\frac{F_{\text {src }}}{\eta G N_{\text {depth }}}+A_{\text {src }} \sigma_{\text {sky }}^{2}+\frac{\kappa\left(A_{\text {src }} \sigma_{\text {sky }}\right)^{2}}{A_{\text {sky }}}},
$$

where $F_{\text {src }}$ is the sky-background-subtracted source flux density or source intensity, $G$ is the detector gain, $N_{\text {depth }}$ is the depth of coverage, $\eta$ is the conversion factor from image-data units to D.N., $A_{\text {src }}$ is the aperture area (in pixels ${ }^{2}$ ), $\sigma_{\text {sky }}$ is the sky background standard deviation (per pixel), $\kappa$ is a factor that depends on the optional background-estimation type or sky model employed in the computation (see below), and $A_{\text {sky }}$ is the area of the sky annulus (in pixels ${ }^{2}$ ). Equation (3) has three terms under the square-root sign. The first two terms are well known: e.g., see Bertin (2006b). The third term accounts for the uncertainty in the background estimation, which SExtractor omits. Equation (3) does not account for possible pixel-to-pixel noise correlations, which are present in co-added images. The derivation of this formula is beyond the scope of this article and may be found in Masci and Laher (2012, in preparation).

Equation (3) allows that the image the user has in hand is not necessarily as observed with the detector's gain factor, but rather the combination of some number of images, as in a co-added image or mosaic. The depth of coverage can vary with source position, and so this must be adjusted for each individual source. The depth of coverage $N_{\text {depth }}$ can be specified on the More Settings panel.

Depending on whether the APT-optional sky average, sky median, or custom sky value is used in the sky background sub- traction, the value of $\kappa$ in equation (3) is equal to either 1.0, $\pi / 2$, or 0.0 , respectively. In the first two cases, APT uses the sky scale, which is defined in $\S 7.4$, as a robust estimator of the sky background standard deviation, $\sigma_{\text {sky }}$; in the latter case, $\sigma_{\text {sky }}$ is taken to be zero. (In this article, robust refers to methods that are generally somewhat less optimal than methods applicable to well-characterized noise, but more stable against outliers. For example, the sample median is a robust estimate of the population mean when the sample is contaminated, but the sample mean is most optimal when the noise is wellbehaved.) In the artificial case of no sky background subtraction (an APT option that is useful in radial-profile interpolation, as discussed in $\S 7.6$ ), only the first term under the squareroot sign in equation (3) determines the source-intensity uncertainty.

The More Settings panel allows the user to specify two additional quantities related to the calculation. It provides for setting an aperture correction, a multiplicative factor greater than 1.0 that, when multiplied by the result for source intensity, accounts for the missing source intensity that becomes a problem when the aperture is too small for the source. This is useful when it is impractical to make the aperture as large as is needed, such as in dense fields. The user can also set a magnitude zero point, so that photometrically calibrated source magnitudes will be computed. The magnitude zero point, if available for the image at hand, comes from prior photometric calibration against reference stars either in the same image or in images acquired by the telescope in the same night (and for the same camera filter used). When the magnitude zero point is determined, it can either be done for the image's exposure time or normalized to an image with a $1 \mathrm{~s}$ exposure time. The case that applies should be documented and, therefore, known. If the latter is the case, then the magnitude zero point for a $1 \mathrm{~s}$ exposure must be corrected for the image's exposure time before inputting to APT, in order to yield accurate results, as APT does not do this automatically. This correction is done by adding $2.5 \log _{10}\left(T_{\exp }\right)$ to the time-normalized magnitude zero point, where $T_{\exp }$ is the exposure time, in seconds.

In the event adjustments are made to the GUI controls that affect any of APT's calculational results, the Recompute Photometry button text will change from the color black to the color red as a reminder to the user that the calculation needs to be updated. This visual device aims to reduce user errors resulting from incorrect associations made between mutually inconsistent inputs and outputs.

BUNIT is a keyword defined by the FITS standard for specifying the physical units of the image data. If this keyword is missing or its setting is null in the image FITS header, then, for clarity in various GUI-panel labels, the software will assume the setting of the default image-data units. This can be changed via the Set Default Image-Data Units option in the Preferences menu, and it is initially D.N. 


\section{SUPPLEMENTARY SOFTWARE FUNCTIONALITY}

\subsection{Image-Data Display}

In the main image-viewing panel, which is located in the upper-right corner of the main GUI panel, the stretch and colortable controls work automatically only for the visible portion of the displayed image, plus some margin around the visible image's edges. This design feature allows the software to run faster and be more responsive. The positions of the panel's scroll bars determine which portion of the image is visible and actively updated when the image-viewing settings are changed. Moving the scroll bars for large images will reveal the once-active portion of the image inside a visually obvious bounding box. The image outside of the bounding box will be displayed with a different stretch and/or color table, which was set earlier in the APT session. To remove the unsightly remnants of the bounding box and refresh the displayed image, two refresh options are available. The Refresh 1 button quickly refreshes just the visible portion of the image to save time. The Refresh 2 button refreshes the entire image by launching multiple processor threads for refreshing the unseen portion of the image, so that immediate GUI control is returned to the user, and additional computer CPU cores, if available on the user's machine, are utilized to finish the job faster. For very large images, however, the threads take some time to complete and may still be running even though the user is allowed to continue normal work with the GUI. To avoid queueing up too many threads, the user is advised to not mouse-click on the Refresh 2 button more than once in a reasonable time interval: at least a few seconds. When needed, the Refresh 2 button text will change from the color black to the color yellow to remind the user to click on this button before scrolling about the image. It is not mandatory for the user to click on the Refresh 2 button when its text turns yellow; the user can defer doing this until after the scroll bars are subsequently moved.

For purposes of image display only, all image-data values outside the interval specified by the stretch extrema are set to the corresponding extreme value. Image-data values that are set to $\mathrm{NaN}$ or Inf are displayed with the blank color set by the user (the color blue is the default). Inf values are handled the same as NaN values, and almost no distinction is made between these two bad-data types. The blank color can be changed using the blank color-picker accessible via the Set ImageDisplay Attributes option in the Preferences menu.

The user can specify the maximum image size that the software will load into memory. This is done using the Set Maximum Image Size option in the Preferences menu. The default maximum image size is 5000 pixels on an image side, and this preference can be reset to as many as 100,000 pixels. For images larger than the preferred value, the user will be prompted to specify the desired portion of the image after its filename has been selected.

\subsection{User Preferences}

Various options in the Preferences menu allow users to change the default settings and then save them to disk for a later APT session. When APT is launched, it automatically loads the preferences from a special file called APT.pref that is located in the invisible subdirectory called .AperturePhotometryTool in the user's home directory. ${ }^{15}$ Users also have the option of later manually loading in another preferences file from a different disk location and filename. If the special preferences file does not exist, then factory-default preferences are loaded into the user's session, but are not automatically saved - the user must explicitly save them via the Save Preferences option in the Preferences menu, if that is what the user wants to do. Within a given APT session, whenever a new primary image is read in, the preferences are restored from the special preferences file. Selecting the Reset Default Preferences option in the Preferences menu will restore the factory-default settings to the user's session, but will not save them (again, the user must manually select the option to do this). If the Save Preferences option is selected, then the current settings of the user's session will be written to a user-selected location and filename, and if that file already exists, then all prior custom settings in that file will be overwritten.

At any time during a user's session, most of the current settings, as set by the various GUI controls within and without the Preferences menu, are the instantaneous user's preferences for the session (albeit not necessarily saved to disk). This can be verified by mouse-clicking on the List Preferences function under the Preferences Menu. One exception is the setting of the aperture geometrical parameters and the centroid and sky annulus inner and outer major radii, whose preferred values should be set via the Set Aperture Size, Shape, and Angle and Set Photometry Size Parameters options in the Preferences menu (these values may be changed to other values directly on the main GUI panel, as needed for experimentation, without affecting the preferences). Therefore, the Save Preferences option will, for the most part, capture the current state of the user's session.

\subsection{Output Files}

During the operation of the software, several output files are created at various stages (see Table 1). With the exceptions noted below, all output files are created in the scratch directory with fixed filenames. The output aperture-photometry-table disk location and filename, the location of the scratch directory, and the user-preferences location and filename can be changed via options available in the Preferences menu. The filename sourcelistByAPT. dat is, by default, generated in the last

\footnotetext{
${ }^{15}$ Note that the environment variable APT_HOME must be set to the location where APT is installed, and this location is not to be confused with the user's home directory.
} 
TABLE 1

OutPut Files CREATED By APT

\begin{tabular}{|c|c|}
\hline Filename & Definition \\
\hline APT.pref & Default filename of customizable user preferences (see $§ 7.2$ ). \\
\hline APT.tbl . & Default filename of output aperture photometry table (see $\S 7.4$ ). \\
\hline fitsHdr.txt $\ldots \ldots \ldots . .$. & Listing of the FITS header. \\
\hline apertureSliceX.dat & Image data corresponding to a horizontal slice across the aperture. \\
\hline apertureSliceY.dat & Image data corresponding to a vertical slice across the aperture. \\
\hline skyScatter.dat .... & Data shown in the sky-scatter graph. \\
\hline sourceScatter.dat & Data shown in the source-scatter graph. \\
\hline curveOfGrowth.dat & Data shown in the curve-of-growth graph. \\
\hline radialProfile.dat $\quad \ldots \ldots \ldots \ldots \ldots$ & Data shown in the radial-profile graph. \\
\hline radialProfileDataFitCurve.dat . & Data-fit curve shown in the radial-profile graph. \\
\hline radialProfileDataFitModel.dat & Data-fit model for aperture interpolation (see $\S 7.6$ ). \\
\hline scatter.dat $\ldots \ldots \ldots \ldots \ldots \ldots$ & Scatter-plot data from user-selected columns in the aperture $\mathrm{p}$ \\
\hline sourceListByAPT.dat $\quad \ldots \ldots \ldots \ldots$ & Source-list file generated by APT's extraction of image sources (see $\S 7.9$ ). \\
\hline
\end{tabular}

directory from which a source-list file was read by the sourcelist tool (see $\S 7.8$ ), or, if this is not available, the scratch directory; there is also the user option on the source-list-creation panel of selecting the location and filename of choice for the newly generated source list (see $§ 7.9$ ).

\subsection{Aperture Photometry Table}

APT generates a table of accumulated results during the course of its normal operation. This is not automatic, however; the user must deliberately mouse-click on the Save Results button after each aperture photometry calculation, in order to write a row of results to the table. Users can save the table to the disk location and filename of their choice. The default location is the invisible subdirectory .AperturePhotometryTool in the user's home directory and the default filename is APT.tbl. Table 2 defines the columns of the table, along with applicable data units, in the order the columns appear in the table. The table can be listed by mouse-clicking on the List Results button located in the lower-left corner of the main GUI panel. The table's data are stored in a plain-text file, which can be easily parsed with a user-supplied script.

\subsection{Graphs}

APT produces three different general categories of graphs. One is simply the aforementioned image histogram, which does not fit into the two remaining categories (see item 5 in $\S 3$, and Fig. 2). Another is a set of different graphs that pertain to the current aperture photometry calculation (category A). The other is the capability of making scatter plots and histograms of userselected columns in the output aperture photometry table (category B). Category-A graphs are most useful for analyzing and refining the current calculation, and category-B graphs are for visualizing a set of calculations, such as might cover a large number of sources extracted from a given image.

There are six different category-A graphs, and these are easily displayed by mouse-clicking on the associated main-GUI- panel buttons located in the middle of the main GUI panel (see item 28 in $\S 3$ ). The choices are Aperture Slice, Curve of Growth, Source Scatter, Sky Scatter, Sky Histogram, and Radial Profile. All of these graphs require that an aperture be overlaid onto the primary image as described above. The graphs may be selected in any order, although the order listed above is a good one for adjusting APT settings systematically for a given source.

There is a text field near the middle of the More Settings panel labeled Default image-data title for the user to specify the graph's image-data title (e.g., Surface brightness). At the bottom of the More Settings panel, there are options that control the data plotted in the category-A graphs. There is a check box labeled Perform image-data conversion that enables the conversion of the image data from the image-data units of the FITS file to any desired source-intensity units. In addition, there are associated text fields for specifying the conversion factor, a string representation of the physical units (e.g., $\mathrm{MJy} \mathrm{sr}^{-1}$ ), and a string representation of the graph image-data title for the converted image data (e.g., flux density). The latter, if the check box is enabled, will override the default image-data title.

Figure 6 shows an Aperture Slice plot generated by APT. The blue and pink curves correspond to slices through the aperture center along horizontal and vertical image axes, respectively. The slices extend across both aperture and sky annulus. Along with the plotted curves are colored lines that visually convey the size and shape of the aperture and sky annulus. All vertical colored lines in the plot map directly to the colors used in the overlay symbol representing the aperture and sky annulus on the image.

Figures 7-10 give random examples of the other categoryA graphs available. The Curve of Growth graph is useful for determining the best aperture and sky annulus major and minor radii (assuming the rotation angle of the elliptical aperture is set correctly). The Source Scatter, Sky Scatter, and Sky Histogram graphs are useful in setting efficacious outlier-rejection 
TABLE 2

Columns in Aperture Photometry Table Generated by APT

\begin{tabular}{|c|c|}
\hline Column(s) & Definition \\
\hline RA_cen, Dec_cen & Sky coordinates of the source centroid (decimal degrees or sexagesimal representation, as set via the Preferences menu). \\
\hline$X, \bar{Y} \quad \ldots \ldots \ldots \ldots$ & Nearest-integer image coordinates of the aperture center (pixels). \\
\hline C_f $\ldots . .$. & $\begin{array}{l}\text { Centroiding flag, equal to } 1 \text { when the option to perform the calculation with the aperture centered on the source centroid has been } \\
\text { selected, and zero otherwise. This option is accessible on the control panel via the More Settings button in the lower-left corner of } \\
\text { the main GUI panel. }\end{array}$ \\
\hline Source_unc & $\begin{array}{l}\text { Uncertainty associated with the source intensity, in data units given by the Data_units column (see below). This calculation uses } \\
\text { Sky_scale as a robust estimator of the sky background standard deviation (see below). }\end{array}$ \\
\hline Data_units & $\begin{array}{l}\text { Data units of the Source_intensity, Source_unc, Sky_median/pix, Sky_average/pix, Sky_RMS/pix, Sky_custom/pix, Sky_sigma and } \\
\text { Sky_scale columns. }\end{array}$ \\
\hline Magnitude & Magnitude associated with the source intensity, after the aperture correction and magnitude zero point have been applied. \\
\hline Mag_unc $\ldots . . .$. & Uncertainty associated with the magnitude. \\
\hline Sky_median/pix & $\begin{array}{l}\text { Per-pixel median of the data in the sky annulus after the sky outliers have been rejected, in data units given by the Data_units column } \\
\text { above. This value, which is a robust estimator of the sky level, times the number of pixels in the aperture form a product that is the } \\
\text { sky contribution optionally subtracted from the integrated image data of the source. }\end{array}$ \\
\hline Sky_average/pix $\ldots . .$. & $\begin{array}{l}\text { Per-pixel average of the data in the sky annulus after the sky outliers have been rejected, in data units given by the Data_units column } \\
\text { above. A disparity between the average and the median is an indicator of skewness in the data distribution. }\end{array}$ \\
\hline Sky_RMS/pix & $\begin{array}{l}\text { Per-pixel rms value of the data in the sky annulus after the sky outliers have been rejected, in data units given by the Data_units column } \\
\text { above. This value is slightly larger than the average, and will be equal to the population standard deviation in the special case of } \\
\text { zero-mean data. }\end{array}$ \\
\hline Sky_custom/pix & Per-pixel custom-sky value specified by the user, in data units given by the Data_units column above. \\
\hline Sky_sigma $\quad . .$. & $\begin{array}{l}\text { Standard deviation of the data in the sky annulus after the sky outliers have been rejected, in data units given by the Data_units column } \\
\text { above, calculated using the unbiased or } 1 /(N-1) \text { method. This result has not been inflated to account for the data-clipping of the } \\
\text { distribution's tails. }\end{array}$ \\
\hline Sky_scale & $\begin{array}{l}\text { Robust estimator of the sky background data dispersion, in data units given by the Data_units column above. It is given by the } \\
\text { difference between the } 84 \text { and } 16 \text { percentiles of the sky-data distribution (which corresponds to approximately } \pm 1 \text { standard } \\
\text { deviation in a Gaussian distribution), divided by } 2 \text {. }\end{array}$ \\
\hline R_o_sky & Major radius of the outer-sky annulus (pixels). \\
\hline N_aper .. & $\begin{array}{l}\text { Number of pixels in the aperture after the source outliers have been rejected (see N_rej column below). It is the data associated with } \\
\text { these pixels that are summed to yield the integrated image data in the aperture. This quantity has a fractional component because the } \\
\text { calculation is done with subpixel resolution. }\end{array}$ \\
\hline N_rej & $\begin{array}{l}\text { Number of source outliers rejected. The rejects represent potential losses of integrated image data, which, as an option, can be filled in } \\
\text { via APT's radial-profile interpolation. }\end{array}$ \\
\hline N_sky & Number of data points in the sky annulus after the sky outliers have been rejected. \\
\hline Image & $\begin{array}{l}\text { Path and filename of the associated image. (Experimental: For Spitzer IRAC images, MJD_OBS, DATE_OBS, pseudo-FILTER } \\
\text { settings are appended after the image filename.) }\end{array}$ \\
\hline
\end{tabular}

thresholds. The example Radial Profile graph is discussed in more detail in $\S 7.6$.

Regarding category-B graphs, selected columns in the aperture photometry table can be plotted via the Plot Results button located in the lower-left corner of the main GUI panel. This button brings up a plot panel that allows the user to customize the graph (see Fig. 11). Both scatter and histogram plots are available. Most of the columns in the aperture photometry table can be plotted against one another in a scatter plot by selecting the desired columns from the pull-down menus. The plot panel controls give flexibility in creating a variety of graphs. A sample scatter plot to illustrate this is given in Figure 12.

JFreeChart, the Java package used by APT to generate graphs, comes with many built-in features for altering the 


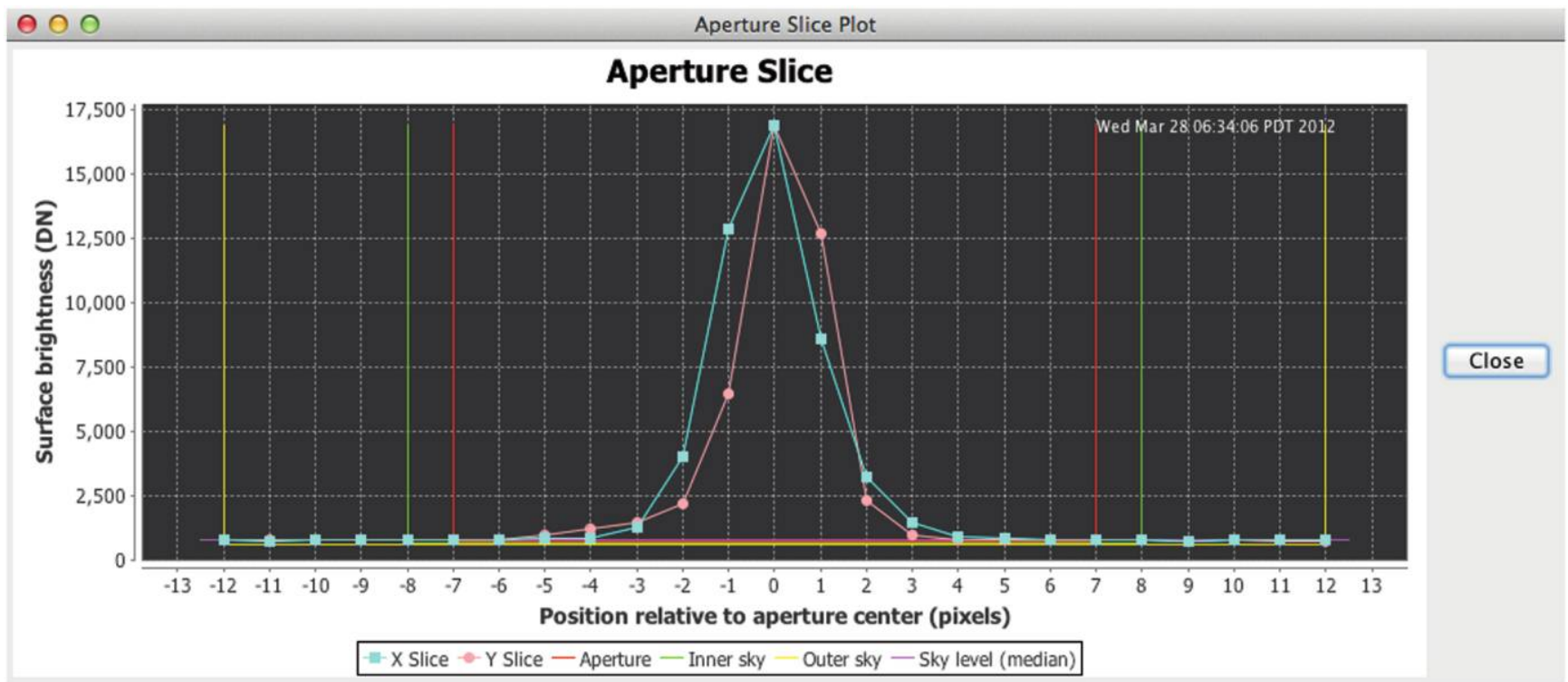

FIG. 6.-Sample aperture-slice plot generated by APT.

graph's appearance. The user can drag the mouse cursor across the graph to narrow the range of the plotted data. The user can also right-button mouse-click on the graph to zoom in and out, change the foreground and background colors, save the graph as a PNG-formatted image, etc. (Mac users will need a mighty mouse and will also have to configure the mouse under the
System Preferences menu to set up right-click as a secondary button.)

The graphing software cannot handle very large or very small numbers and sometimes responds by creating a graph with no numbers on the affected graph axis. If this is encountered, adjust the outlier-rejection range as needed until the problem goes

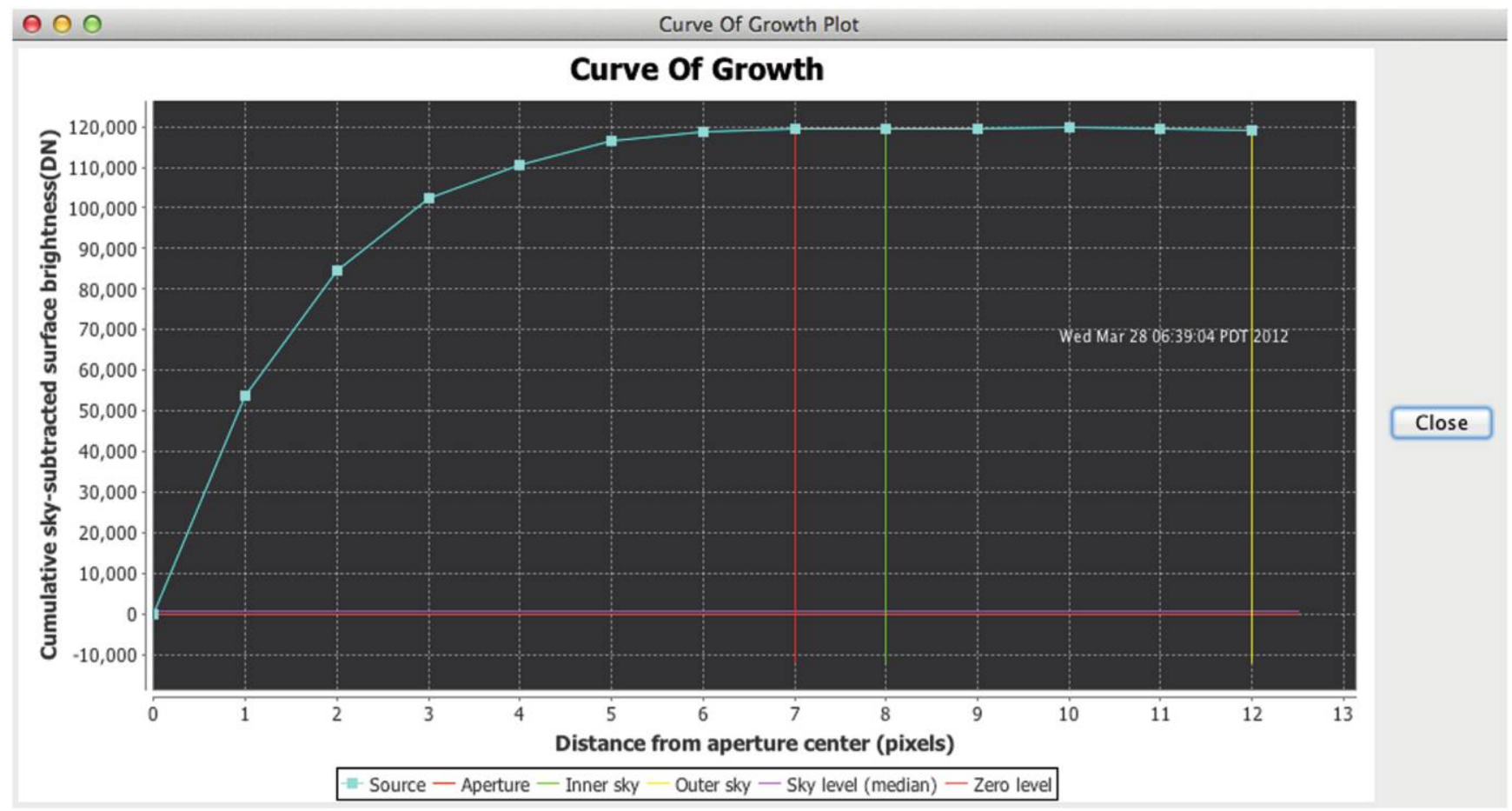

FIG. 7.-Sample curve-of-growth plot generated by APT. 


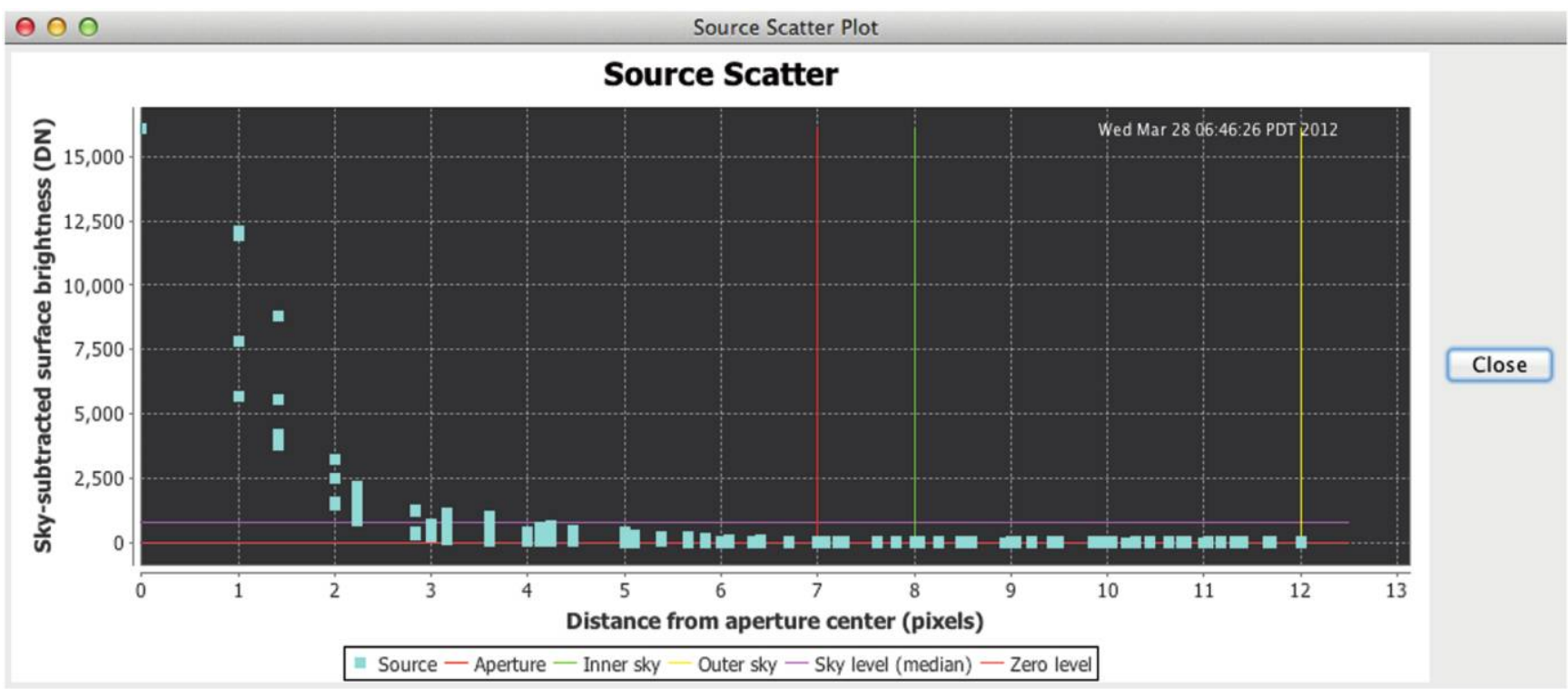

FIG. 8.-Sample source-scatter plot generated by APT.

away (click on the More Settings button on the lower-left side of the main GUI panel to bring up the outlier-rejection controls).

Each time a graph-generation button is mouse-clicked, a new graph is displayed, and previously displayed graphs are not destroyed. We deliberately made the choice of allowing users to display an arbitrary number of graphs on their computer screens to enhance APT's function as an educational tool. This software behavior allows users to create and compare graphs from different APT settings. Users must exercise some discipline in closing graph windows themselves, in order to prevent the proliferation of graph panels on their computer screens. The date and time label on each graph is helpful for differentiating the on-screen graphs.

\subsection{Radial-Profile Interpolation}

APT's radial-profile-graph panel has extra controls for fitting a parameterized radial-profile model to the data associated with the currently selected source (see Fig. 13). The radial-profile model is a smooth, continuous curve that is designed for a radially symmetric source:

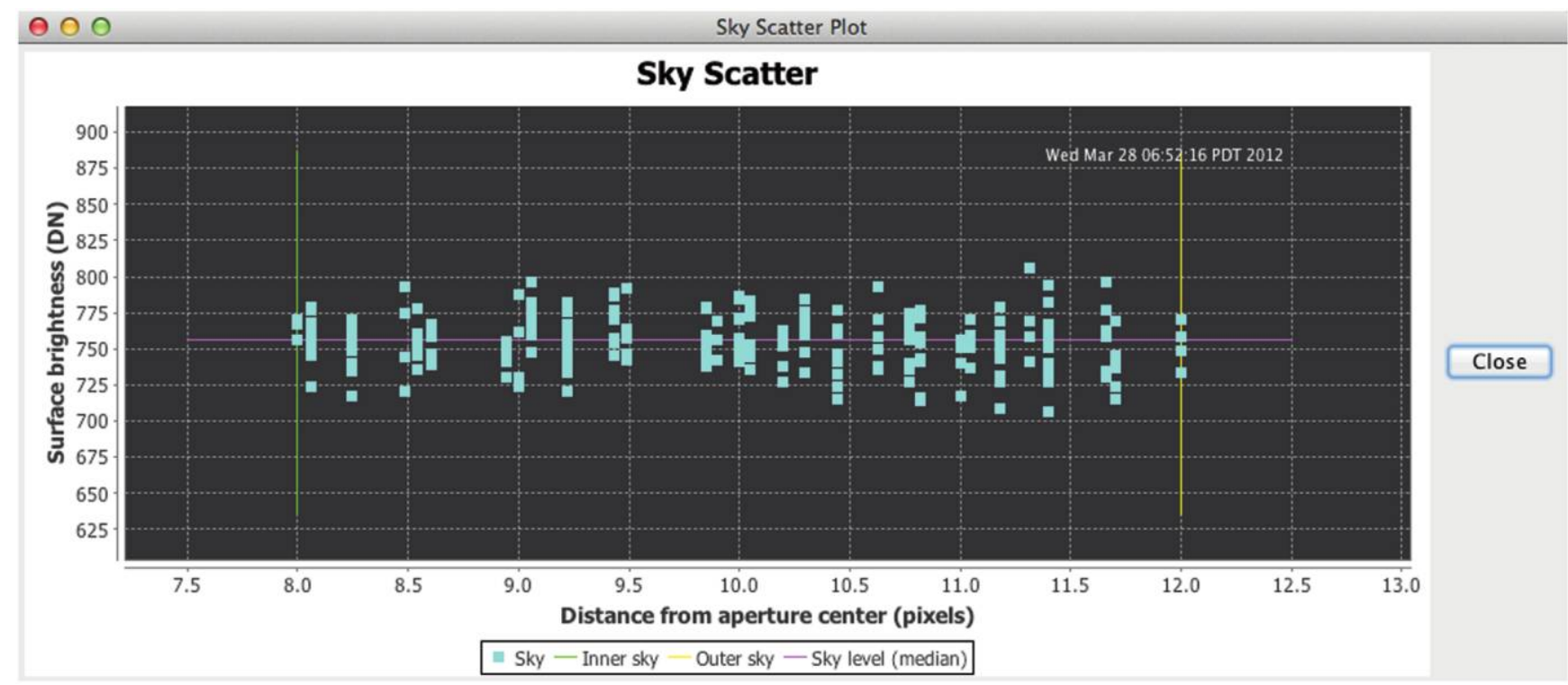

FIG. 9.-Sample sky-scatter plot generated by APT. 


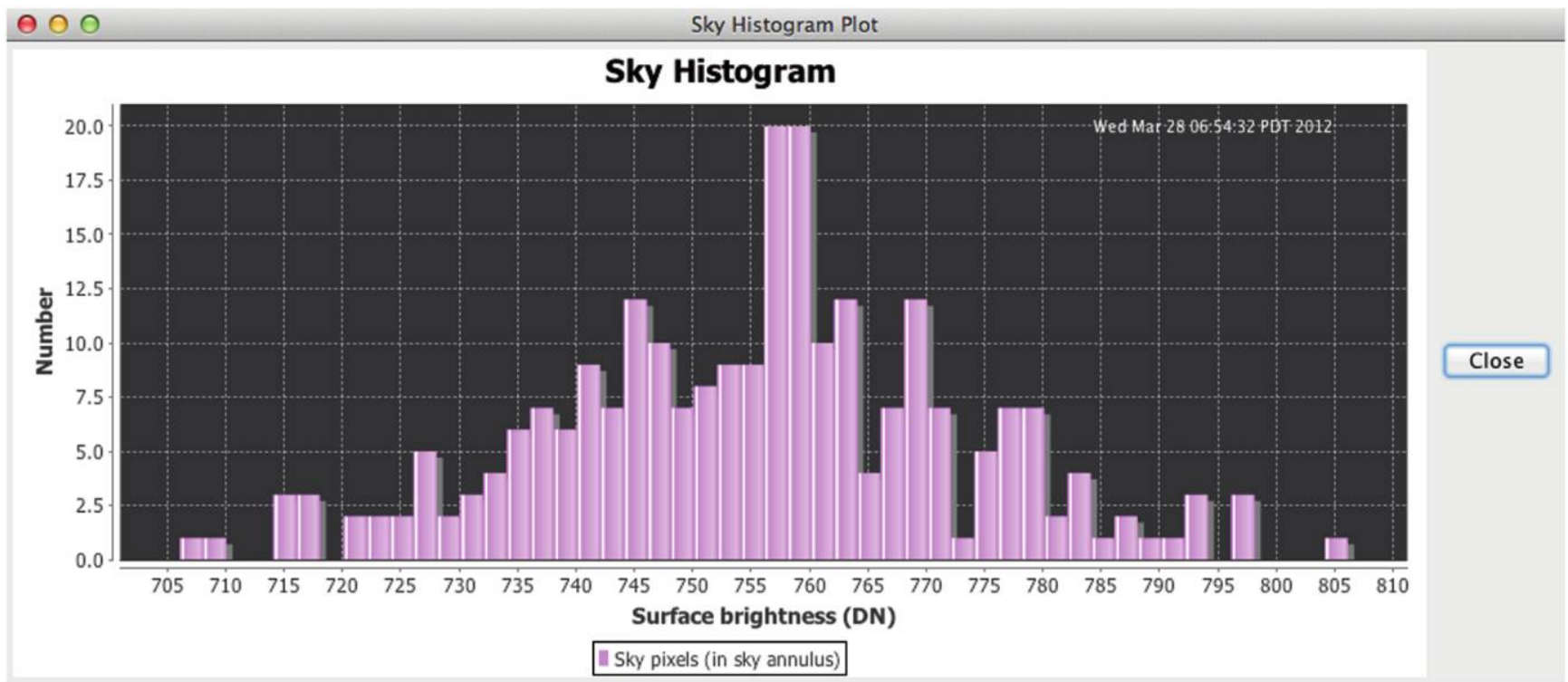

FIG. 10.-Sample sky-histogram plot generated by APT.

$$
S(r)=A+B r+C r^{2}+D r^{3}+E r^{4}+F \exp \left(\frac{-r^{2}}{2 \sigma^{2}}\right),
$$

where $S(r)$ is the pixel intensity or surface brightness as a function of radial distance from the aperture center $r$, in pixels, which is constrained by $r \geq 0$; the data-fit coefficients $A$ through $F$ are determined via linear regression; and $\sigma$ is a fixed parameter that can be manually adjusted to optimize the data fit. The rightmost term containing the exponential function is a scaled Gaussian distribution, and $\sigma$, therefore, is the familiar measure of the distribution's half-width. This model was deliberately chosen to be relatively simple and yet have enough degrees of freedom to work well on images with a point-spread function (PSF) that is by-and-large radially symmetric. Optimizing the data fit involves manually choosing the value of $\sigma$ that minimizes the leastsquared-error goodness-of-fit measure $\chi^{2}$.

In order to build a model for the image of interest, the user must adhere to the following instructions precisely:

1. On the More Settings panel (accessed via the More Settings button on the lower-left part of the main GUI panel), select the Model 0 source algorithm and the Model A sky algorithm. Also, the check box labeled Perform new image-data conversion on the More Settings panel should be unchecked. A picture of the More Settings panel is shown in Figure 5. Warnings will be given when the model is saved with these conditions not met.

2. Select a source from which to create the radial-profile model, and overlay an aperture onto it in the main image-viewing panel. For a model that is to be representative of the sources in the image, it is best to select a moderately bright, unsaturated source, which will be photon-noise-dominated and have a relatively lower percentage of noise versus signal.
3. Fit the radial-profile data by operating the controls on the right-hand side of the radial-profile-plot panel. A new fit will be done when the user changes the value of $\sigma$, and this is reflected immediately by the software displaying the resulting data-fit curve in the radial-profile graph. The text field to the left of the Reset Sigma button can be filled in with an initial guess. The Reset Sigma button causes the data fit to be done with the value of $\sigma$ in the text field. The slider control below it causes data fits to be done with values in the neighborhood of the textfield value of $\sigma$. Clicking on the Reset Sigma button after varying the slider will revert $\sigma$ of the data fit to the initial guess and center the slider. The Set Slider button centers the slider on the current value of $\sigma$ and puts that value in the text field as the current initial guess. The Recompute Fit button performs the same operations, plus does the data fit one more time for good measure. Typically, the best fit is obtained when $\chi^{2}$ is minimized: that is the goal of the linear regression as implemented in APT. Hint: A not-so-good fit can sometimes be made better by reducing the aperture size and sky annulus major radii. When a new fit is done, the scale and offset parameters are reset to 1.0 and 0.0 , respectively (more details about these model-exogenous parameters are given below).

4. Once the user has obtained an acceptable fit, the model parameters can be saved by mouse-clicking on the Save button. The model parameters are written to a file in the scratch directory called radialProfileDataFitModel.dat. Only the data-fit model that has been written to this file will be subsequently used in APT calculations involving interpolation.

After the radial-profile model parameters have been saved (as described in the above steps), the model can be subsequently used on any source in the image for interpolation with any 


\begin{tabular}{|c|c|c|}
\hline \multicolumn{3}{|c|}{ Plot Photometry Data } \\
\hline X plot size (pixels): & 900 & \\
\hline Y plot size (pixels): & 700 & \\
\hline Plot title: & from Ape & e Photometry Tool \\
\hline $\mathrm{X}$-axis title: & Source int & ity (D.N.) \\
\hline Y-axis title: & arce-inten & uncertainty (D.N.) \\
\hline $\mathrm{X}$-axis data: & Source_i & nsity \\
\hline Y-axis data: & Source_L & $\doteqdot$ \\
\hline $\mathrm{X}$-axis minimum: & 0 & \\
\hline $\mathrm{X}$-axis maximum: & 500000 & \\
\hline Y-axis minimum: & 200 & \\
\hline $\mathrm{Y}$-axis maximum: & 1200 & \\
\hline $\mathrm{X}$-axis number format: & $0.0 \mathrm{EO}^{2}$ & \\
\hline $\mathrm{Y}$-axis number format: & 0 & \\
\hline X-axis type: & (- Linear & Logarithmic \\
\hline $\mathrm{Y}$-axis type: & $\odot$ Linear & Logarithmic \\
\hline Invert plot? & $\square$ & \\
\hline Number of histogram bins: & 50 & \\
\hline Scatter Plot & Im Plot & Close Window \\
\hline
\end{tabular}

FIG. 11.-APT's control panel for plotting results.

of the appropriate source models available and any of the available sky models (this, of course, assumes that the image data are sufficiently sampled; in the case of data undersampling, one cannot reconstruct a radial profile with goodenough accuracy). The models can be selected on the aforementioned More Settings panel. The source models that involve interpolation are described as follows.

The model 1 source model is used for interpolating across aperture pixels with $\mathrm{NaN}$ values or Inf values, in order to fill in the missing source-intensity contributions. It also replaces the missing source intensity associated with rejected outliers in the aperture, provided that the interpolated result falls within the lower and upper limits set for source-outlier rejection. Temporarily zapped pixels are also interpolated (see $§ 7.7$ ).

The model 2 source model is used for interpolating source intensities in all aperture pixels, even for those pixels with good data. This model will thus yield a smoothed-data result.

When either of the source models involving interpolation is selected, a two-parameter linear regression is automatically performed for each calculation to scale and offset the radial-profile model (which is treated as a fixed model here), in order to best fit the model to the data for the sources of interest. Thus, the

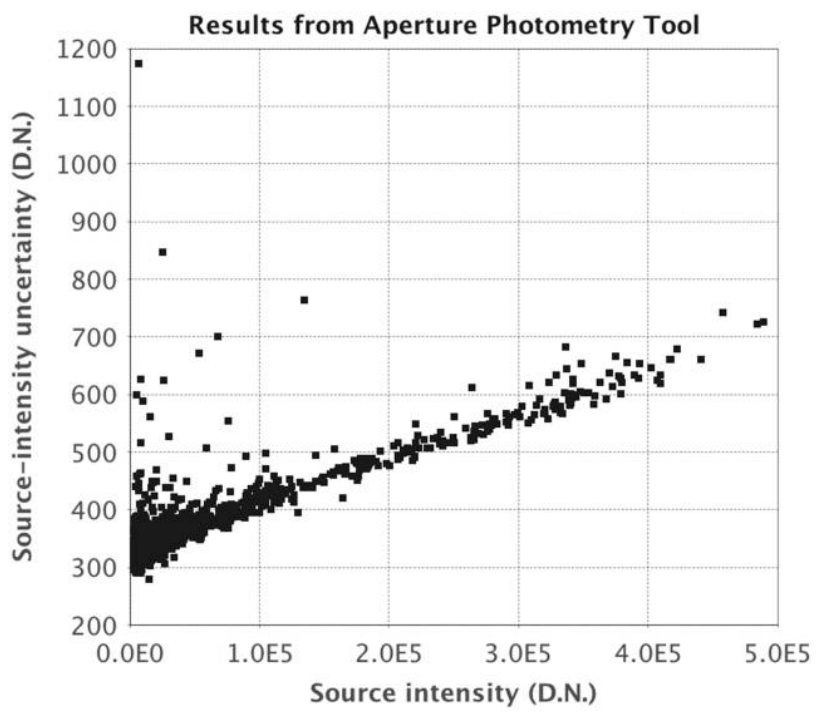

FIG. 12.-Sample scatter plot of results generated by APT.

radial-profile model can be derived from one source and applied to other sources. This model is particularly useful when the image data have a radially symmetrical PSF and the PSF is invariant over the image. The results that are computed with radial-profile interpolation will be less sensitive to the effects of noise in the image data. However, for saturated sources with missing data at small $r$, and especially at $r=0$, extrapolation of the radial-profile model essentially occurs through the computation of the scale and offset, and this can lead to potentially large errors in the results for such cases.

The radial-profile graph panel has also been equipped with controls for manually scaling and offsetting the radial-profile model (which are located at the bottom of the panel). The current purpose of these controls is mainly to allow the user to experiment with the scale and offset parameters and to instantly visualize the effects on the plotted model curve.

\subsection{Pick/Zap Tool}

The pick/zap tool, which is accessed via the Pick/Zap button in the bottom-middle portion of the main GUI panel (see Fig. 14), has a few useful functions for probing and fine-tuning aperture photometry calculations. After an aperture has been overlaid onto the primary-image source, an enlarged subimage centered on the aperture is displayed in the pick/zap tool's work area. Moving the mouse cursor over the subimage causes the passed-over pixel positions and associated image-data values to be rapidly displayed.

When the Pick radio button is selected (and the subimage is displayed), the user can mouse-click on the subimage to pick out specific pixels. Picked pixels are highlighted in the subimage by an outline around the pixel in the current pick color. The picked locations and associated image-data values are listed 


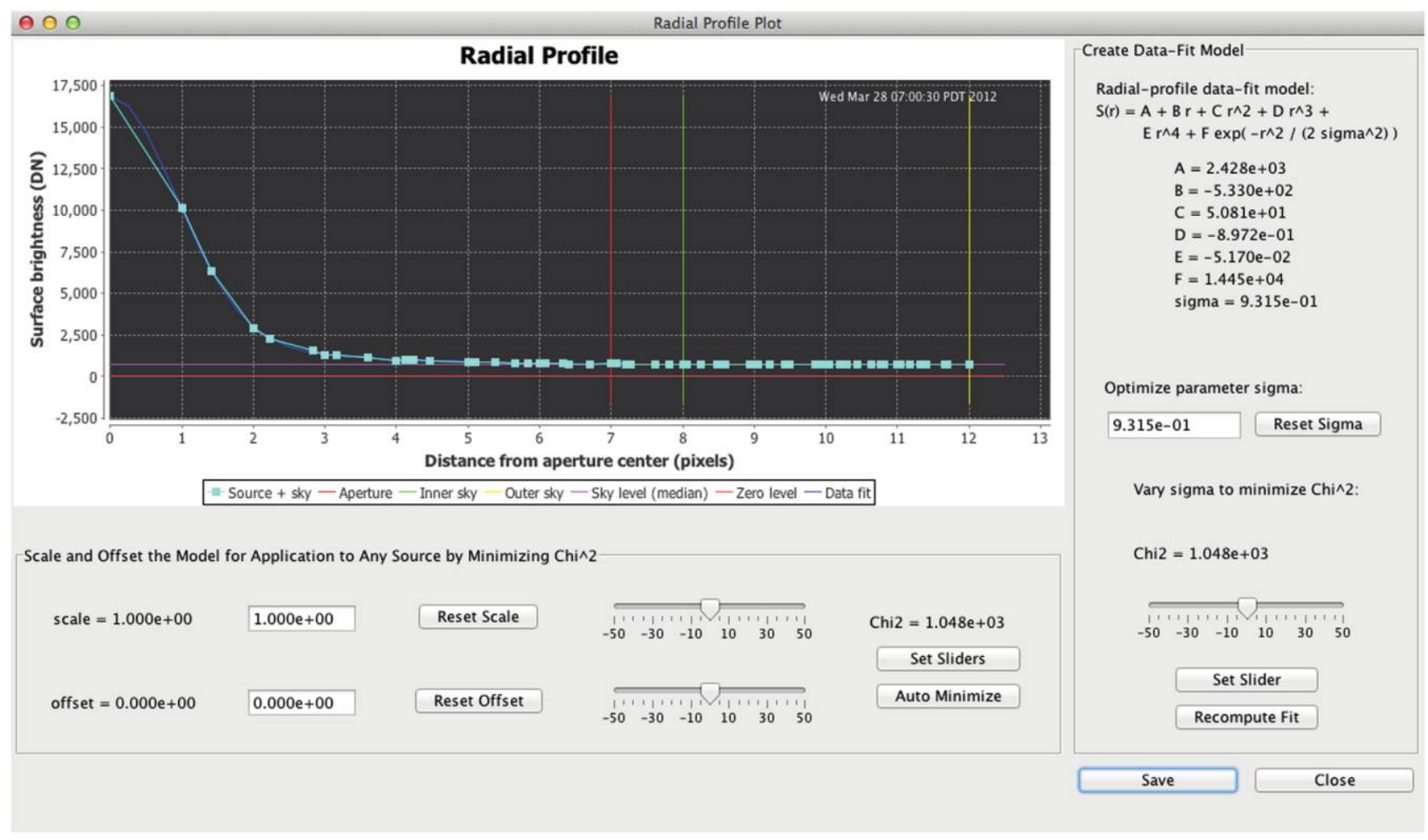

FIG. 13.-APT's radial-profile panel. The example data fit shown has been optimized.

in the adjacent uneditable text area. The pick color can be changed via the Set Image-Display Attributes: Pick Color option in the Preferences menu.

With the Zap radio button, the user can temporarily set specific pixel values to NaN by mouse-clicking on the pixels of interest. NaN values are excluded from the calculations, but this happens only after the Recompute Photometry button has been clicked to update the calculation. The pixel-zapping functionality is useful, for example, for eliminating sources from the sky annulus that adversely affect the sky background calculation. Zapped pixels are displayed in the subimage with the blank color, and their locations and associated image-data values are listed in the adjacent text area. The blank color can be changed via the Set Image-Display Attributes: Blank Color option in the Preferences menu.

The Hide/Show Aperture button is a toggle that alternately hides and shows the overlaid aperture. It is included as a convenience for more clearly showing the subimage. When the aperture is not hidden, all aperture pixels are outlined in the color red on the subimage, except for the aperture-center pixel, which is outlined in the color cyan to indicate the aperture center. The sky annulus pixels are outlined in the color green. The outlined aperture pixels are complete pixels and do not reflect that the aperture photometry is computed at subpixel resolution.

The picks and temporary zaps are kept on the subimage and in the text areas when the Hide/Show Aperture button is pressed. This is also true when any of the image-display controls are adjusted, including stretch, linear/logarithmic scaling, color table, and pick and blank colors. The aperture geometrical parameters and the centroid and sky annulus major radii can also be changed without clearing the picks and zaps. The same goes for changes made within the More Settings panel. After these sorts of changes, in which the aperture may be resized, but may not be moved, either the Recompute Photometry button text will change from the color black to the color red to indicate that the calculation needs to be updated or it will be automatically recalculated in the case of major radii changes where the user hits the Enter key after the change. Clicking on the Recompute Photometry button will nevertheless use the new settings to update the calculation.

Finally, any adjustment that changes the aperture's position will cause the picks and temporary zaps to be cleared, and the image-data values will revert to those in the original image. The software behaves this way to inculcate the understanding that the zaps are temporary. The picks and zaps are cleared when the aperture is moved, because it is presumed that the user is done with the calculation and ready to do another. We also made this design choice to prevent the frustration of a subsequent proximate calculation. Note that $\mathrm{NaN}$ values in the original image will, of course, be preserved. The aperture can be moved by mouse-clicking on either another source in the main imageviewing window or the Snap button, which will automatically 


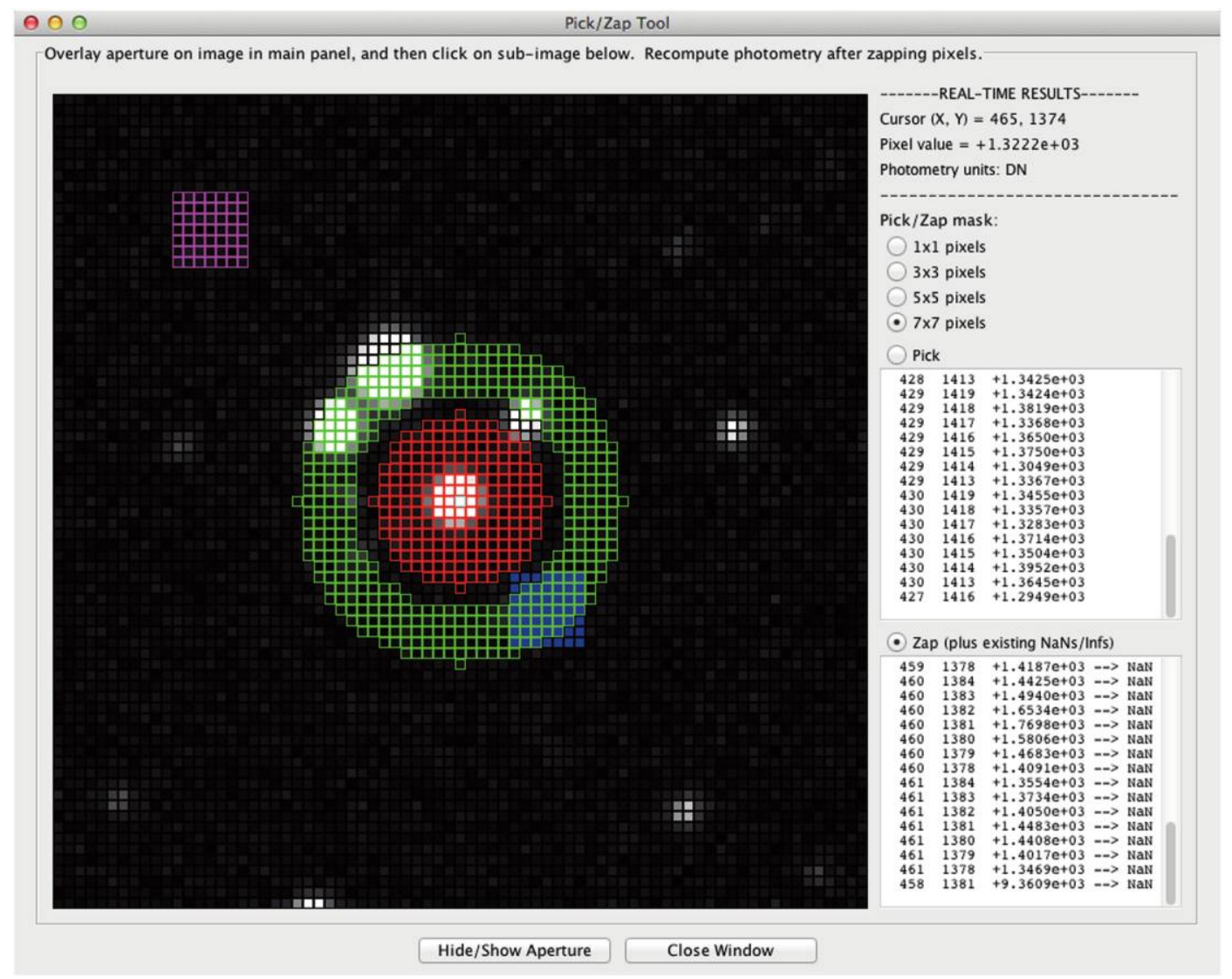

FIG. 14.-APT's pick/zap tool. The picks in a $7 \times 7$ block of pixels are shown in magenta. The zaps in a block of the same size are shown in blue.

move the aperture to the centroid position. Adjusting the aperture-position spinner controls will also clear the picks and zaps, although the aperture movement itself, in this case, will not be done until the Recompute Photometry button has been utilized. Clicking on the frame around the main image-viewing window when the mouse cursor is shown in the crosshair style will cause the overlaid aperture and displayed subimage to be removed, as well as the picks and zaps.

\subsection{Source-List Tool}

After an image for aperture photometry calculations has been selected, the user can access the source-list tool via the Source List button near the upper-left corner of the main GUI panel. Figure 15 shows the tool's user interface. The tool does automatic or manual processing of a source list associated with the chosen image.

An input source-list file is required for the tool, and the file can be created in a variety of ways. It can be created by parsing the output of popular source-extraction software, such as SExtractor, with a user-provided script. It is possible to create a source list by hand-editing or parsing with a script APT's output aperture-photometry-table file from an earlier session (after copying it to another filename). In these cases, the Choose
Source List button is utilized to read in an existing source-list file. Alternatively, APT has functionality for fast, automatic source-list creation by extracting sources from the current primary image directly and then generating a source-list file. The Create Source List button is utilized for this purpose (see § 7.9).

The format of the source-list file is plain text and consists of one set of source coordinates per line in the file, where the coordinates are delimited only by one or more spaces. Either image-pixel coordinates or equatorial coordinates are permitted. Equatorial coordinates, if given, must be converted into decimal degrees. Here is a short example source-list file that gives the pixel positions of source centroids:

$723.2 \quad 279.0$

729.4145 .45

622.195205 .857

595.9318 .4

731.11195 .54

674.84140 .09

643.2153 .1

735.0161 .6

A "file improperly formatted" error will be issued if the file format is incorrect (e.g., comma-separated values are not 


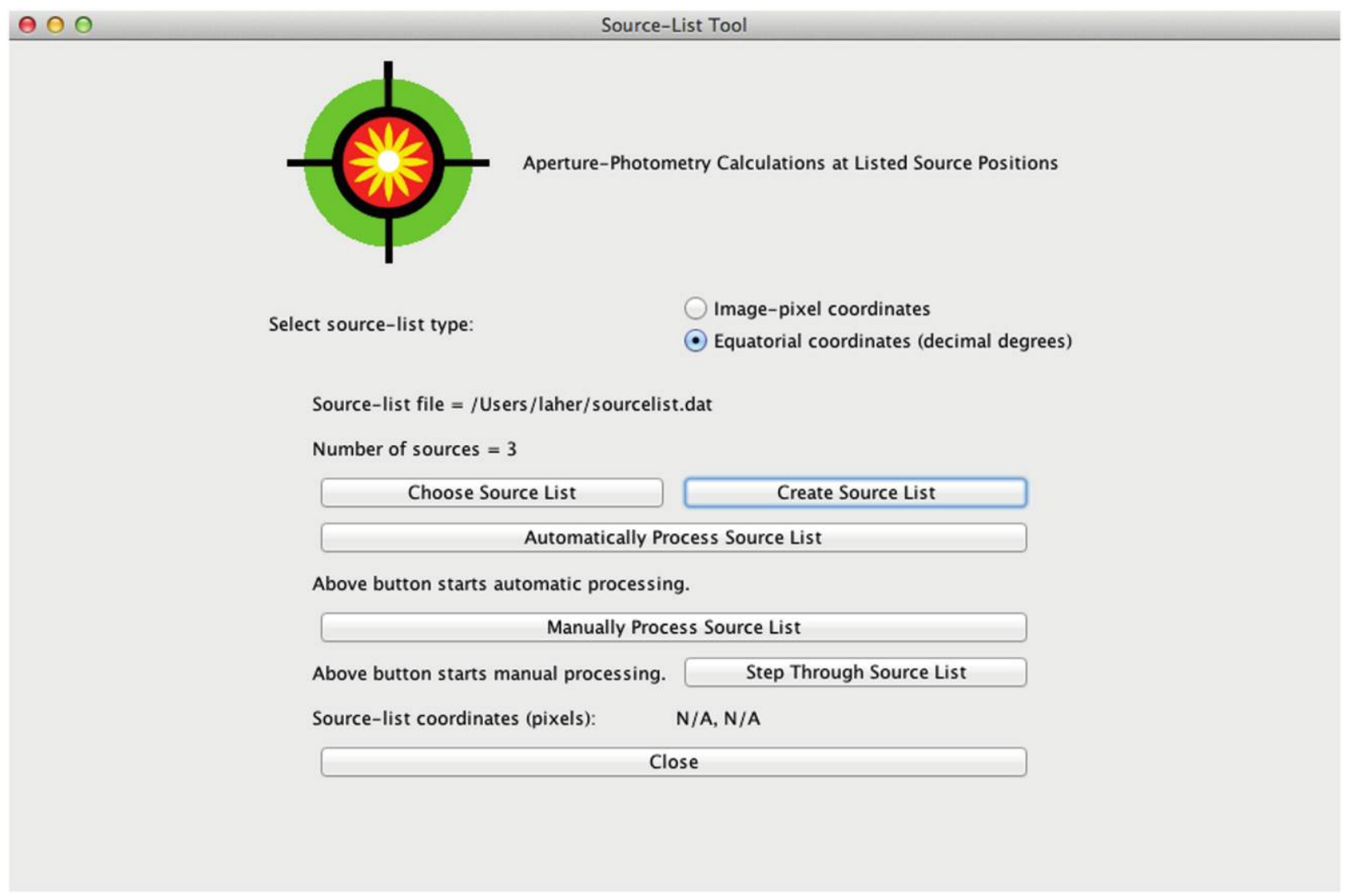

FIG. 15.-APT's source-list tool for performing aperture photometry calculations from a source list.

allowed). The maximum number of sources per source-list file allowed by APT is 100,000 . A set of radio buttons for the user to indicate the source-list-coordinates type is available on the source-list-tool panel.

The user has the option of selecting the button labeled Automatically Process Source List, in which case calculations will be performed for all sources in the source list for the current aperture photometry settings. Thus, it is important that the user select the desired settings before mouse-clicking on this button. Once this option has been selected, identical calculations will be done for all sources in the source list, and a record will be automatically saved for each source in the aperture-photometrytable file. The marker Automatic Source-List Processing ... will be written to the aperture-photometry-table file prior to saving the aperture-photometry records.

Another option available to the user is the Manually Process Source List button, in which case an aperture will be overlaid onto the image for the first source in the source list, and the message "Ready to process source number 1" will be displayed. The user can, at this time, manually adjust any or all of the aperture photometry settings for that particular source on the main GUI panel and via the More Settings button and then press the Recompute Photometry button and view the graphs to make sure that the settings are just right. After the user is satisfied with the settings for that particular source, the Step Through Source List button can be pressed, which will redo the calculation, save a record of the results to the aperture-photometrytable file, and then overlay an aperture onto the image for the next source in the source list. The marker Manual Source-List Processing ... will be written to the file prior to saving the results record for the first source. The above steps are repeated for each successive source in the source list. After the source list has been exhausted, the message "Done with source list." will be displayed. To reset manual processing and start again (perhaps after another selection of aperture photometry settings have been made), simply press the Manually Process Source List button once more.

\subsection{Source-List Generation}

The Create Source List button on the source-list-tool panel pops up another panel with functionality to generate a source list by extracting sources from the current primary image (see Fig. 16) and to then write the source list to a file. The source list is generated with image-pixel coordinates. Users can either save the source list to the disk location and filename of their choice using the Reset Source-List Filename button on the 


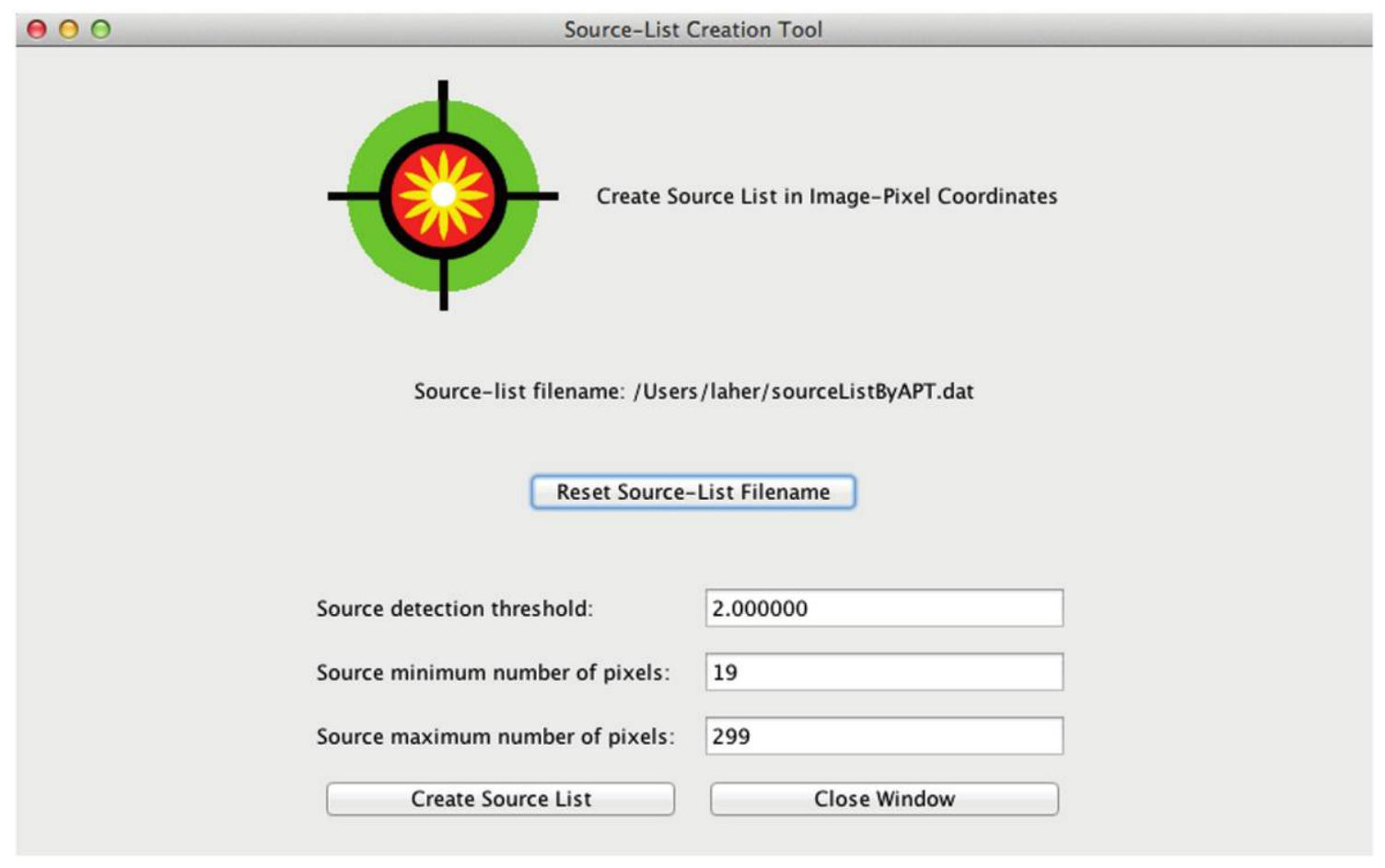

FIG. 16.-APT's source-list creation tool.

panel, or they can keep the default location and filename (see $\S 7.3$ ).

There are three options for controlling the source-list generation. The Source-List Creation Tool panel has text fields for three input parameters: source-detection threshold and minimum and maximum numbers of contiguous pixels allowed for a source. The source-detection threshold is specified in number of datadistribution half-widths. It is used to calculate the image-data threshold at each image pixel position. The image-data threshold is defined as the local background median plus the sourcedetection threshold times the global dispersion of the image data. The dispersion is computed by taking one-half of the difference between the 84 and 16 percentiles. The two remaining parameters control the size range the user allows for detected sources. The source minimum number of pixels is a parameter used to exclude sources with an insufficient number of detected pixels above the image-data threshold. The parameter for the source maximum number of pixels is useful for excluding very large sources that may lie outside of the user's sphere of interest. A source-detection threshold of at least 1.0 is recommended. Either setting the threshold too low or not narrowing the source size range enough may result in more detections than the software can handle, in which case the software will warn the user and recommend another try with a higher threshold. Up to 100,000 segmented detections are currently allowed by the software, where the segments consist of 100 pixels each.

The algorithm for computing the local background median is given as follows. The primary image is divided up into
$N \times N$ pixel cells, where $N$ is an odd number that varies with the size of the image, is chosen to have at least 10 cells across the shortest image dimension, and is allowed to be no smaller than 33 pixels. The median of the image data in each cell is computed, and bilinear interpolation over the cells is then used to estimate the spatially varying local background median. Special handling of the calculation is done at the far image edges to avoid extrapolation.

Source detections occur for image-data pixels that are both greater than or equal to the spatially varying image-data threshold and greater than zero. A source is defined by a set of contiguous detections, where contiguous pixels are joined either at pixel sides or corners. The centroid of each source defines its position in the image, and the centroid image coordinates are written to the specified source-list file.

\subsection{Simple-Photometric-Calibration Tool}

The software includes a user-friendly tool to provide the capability of performing simple photometric calibration (see Fig. 17). The tool matches two source lists and computes a single parameter, the photometric zero point, via sum-of-squared-error minimization. At least one match is required, but no more than 100,000 matches are allowed in the computation. The algorithm makes two passes through the data. The first pass computes robust data statistics: namely, the median and the data dispersion given by one-half of the difference between the 84.1 and 15.9 percentiles. The second pass applies outlier rejection and faint-source rejection to filter the data. A sufficient variety of 


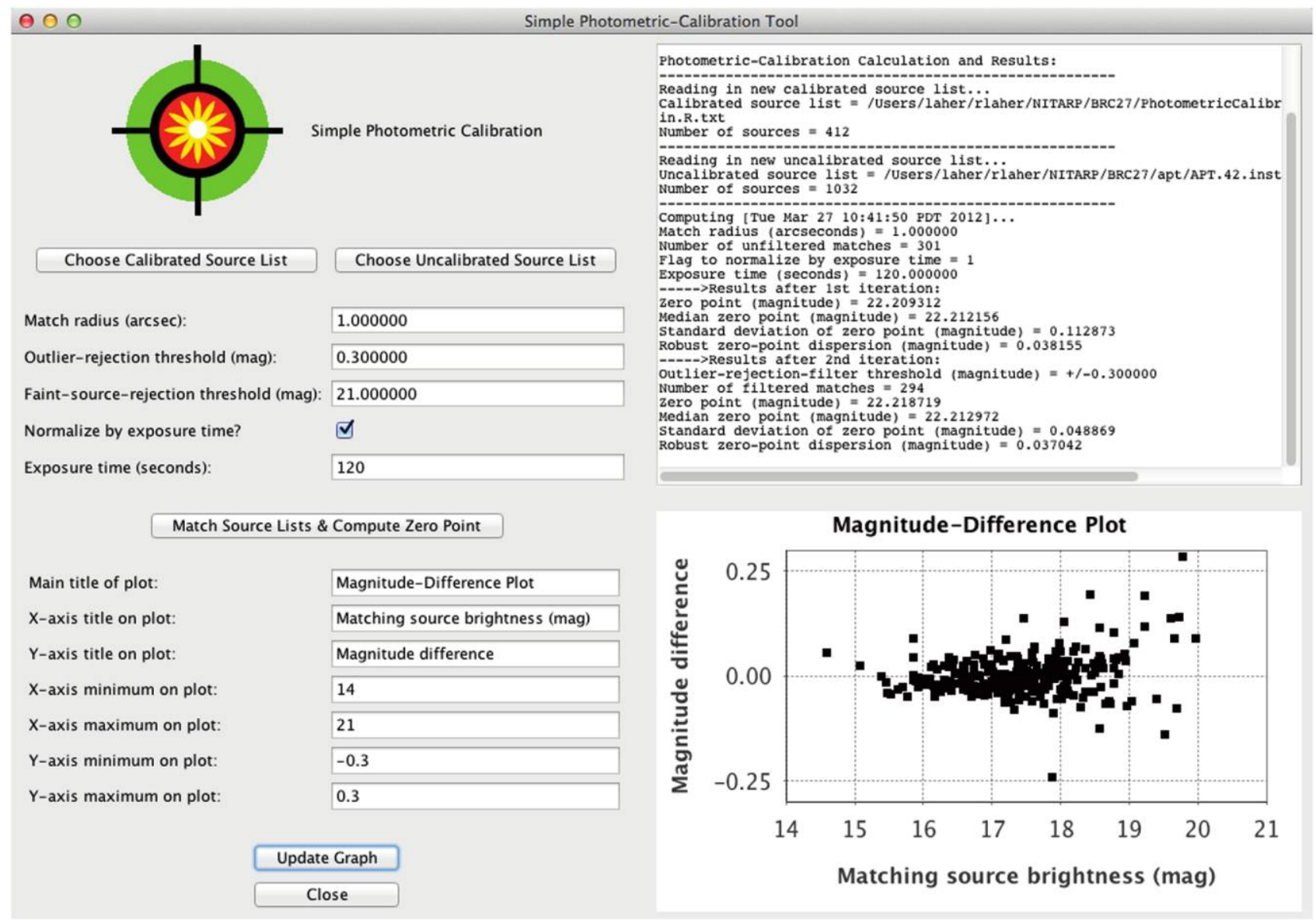

FIG. 17.-APT's simple-photometric-calibration tool.

data statistics are outputted to guide the user in setting up repeat calculations to refine the result. These include the numbers of sources in each list; the match radius (arcseconds); the exposure-time-normalization flag; the numbers of unfiltered and filtered matches; the outlier-rejection-filter threshold for the magnitude difference (magnitude); and the first- and secondpass mean and median zero points, standard deviation, and aforementioned robust data dispersion (all in units of magnitude).

The required inputs are two space-delimited source lists in plain-text files. The software assumes that one of the source lists is calibrated and the other is not. The calibrated source list must have only the three following columns: right ascension, declination, and absolute magnitude. The uncalibrated source list must have only the three following columns: right ascension, declination, and instrumental magnitude. The equatorial coordinates must be in decimal degrees. Each source-list file may include a header line containing three space-separated, singleword column titles (e.g., RA Dec Mag). The software will read in up to 100,000 sources from each source list. The source lists are, by default, expected to be located in the source-list directory specified in the APT user preferences, but the user can navigate elsewhere, if necessary.
There are optional inputs to control the computation. The match radius gives the maximum tolerable distance between sources for a match, and its default value is $1^{\prime \prime}$. The double-sided outlier-rejection threshold, in magnitude, allows outliers to be defined and excluded from the computation, and its default value is 0.3 . The faint-source-rejection threshold, in magnitude, assures that only matches of sufficient brightness will be included in the computation, and its default value is 21 . The tool will optionally normalize the zero point by an input exposure time, and the default exposure time is $1 \mathrm{~s}$ (which is equivalent to not normalizing by the exposure time).

The tool generates and displays a plot of magnitude difference versus magnitude. There are options to customize the plot on the tool's main panel. The user can also right-click on the plot to expose additional plotting options. One of these options allows the user to save the plot as a PNG image.

\subsection{Image Comparator and Blink}

APT comes with a pop-up tool, called the Image Comparator, for comparing images side by side. The image comparator is basically a panel containing a series of up to four magnifier tools 


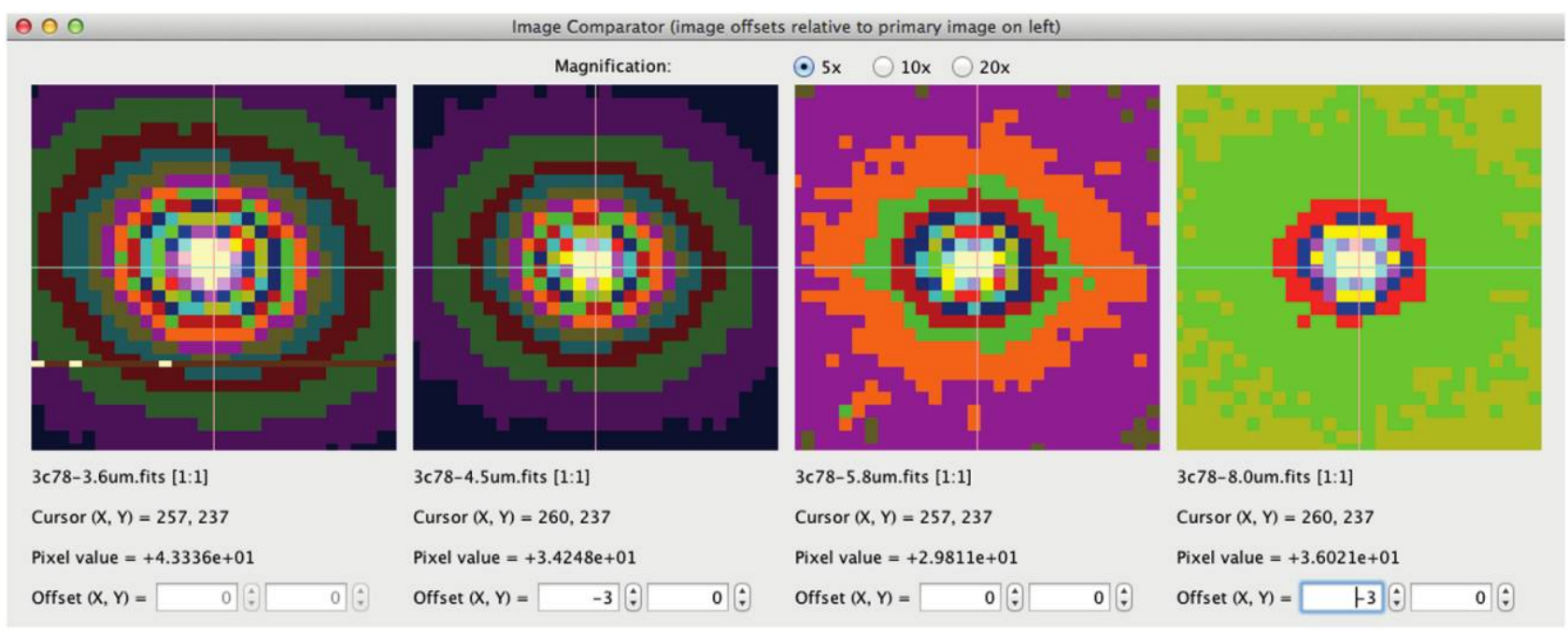

FIG. 18.-APT's Image Comparator showing Spitzer IRAC images in the 3.6, 4.5, 5.8, and $8.0 \mu \mathrm{m}$ bands. The primary image is on the left.

that enable simultaneous comparison of the primary image with up to three different comparator images (see Fig. 18). This functionality is accessed via the Image Comparator button located in the upper-middle portion of the main GUI panel. The comparator images can be offset in $x$ and $y$ relative to the primary image using the available spinner adjustments, if necessary. Separate control over the stretch of each comparator image is currently not implemented. For comparing images with drastically different stretches, judicious use of the primary-image logarithmic-stretch capability can compensate somewhat for this tool's deficiency.

Closely related to the functionality of the Image Comparator is APT's image blink capability. Repeatedly mouse-clicking on the Blink button located in the lower-middle portion of the main GUI panel will quickly cycle the image display from the primary image to the first comparator image in the sequence, subsequently advancing to the next comparator image, if available, and then eventually back again to the primary image. This capability is good for spotting image pixels with variable stars or affected by cosmic rays.

\subsection{Batch Mode}

There are two different options available for running APT in batch mode. The first is that APT can be executed with an image specified on the command line, in which case that image will be loaded and displayed in the GUI:

APT.csh image.fits

The second option is that APT can be executed with both an image and source list specified on the command line, in which case the image and source list will be loaded by the software and processed by APT's source-list tool, without displaying the GUI:

APT.csh image.fits source.lst

This noninteractive batch-mode command is handy for generating an aperture-photometry-table file for the source list from a script. Control is returned to the user's command shell after APT terminates. The image file must be specified before the source-list file. The parameters that control how the source list is processed by the source-list tool are specified by running APT beforehand and saving the parameters as user preferences in the default location (which is loaded automatically upon APT startup).

\subsection{Internationalization}

We implemented a simple workaround for the sticky problem of commas used in place of decimal points on computers in some non-US countries. The APT startup scripts (APT.CSh and APT.bat) force English as the user's language and the United States as the user's geographical region when invoking the JVM. This is done using the following JVM options:

-Duser. language=en -Duser.region=US

The net effect is to require the American usage for input floating-point numbers (e.g., in FITS headers) and to ensure the same for any output floating-point numbers (e.g., written to the aperture-photometry-table file), regardless of the language selected when a computer's operating system was installed. The workaround was verified for an APT installation on a German Windows machine. 


\section{SOFTWARE LIMITATIONS}

Some of the functionality described above is admittedly not well integrated into the main functionality, and these areas could use more work. For example, the Simple-PhotometricCalibration Tool could be upgraded to allow APT's output aperture photometry table as an input (see $\S 7.10$ ). Below is a list of items that could or will be addressed in later software versions (in no particular order). Future software status and updates will be posted on the APT World Wide Web site.

1. Handle accompanying image-data masks and uncertainty images.

2. Account for sky background variation across the aperture in the calculation.

3. Change the number of standard deviations used in outlier rejection for sky background estimation from a hard-coded value into a user-specifiable parameter.

4. Inflate the sky background standard deviation after rejecting outliers, in order to account for the clipping of the distribution's tails.

5. Include pixel-to-pixel noise correlations and spatially varying co-add coverage depth in the calculation of sourceintensity uncertainties.

6. Implement bit flags to indicate potential problems related to the calculation; e.g., source saturation, source located near edge, source in crowded field, etc.; Cf. SExtractor (Bertin \& Arnouts 1996).

7. Upgrade the image comparator to have independent stretch controls and automatic image registration using the images' WCS solutions.

8. Add functionality to compute a suite of statistics for a given column of data in the aperture photometry table.

9. Add zoom-in/out capability for the main imageviewing panel.

10. Add a button that rotates an image so that north is up, based on the WCS in the FITS headers.

11. Add functionality to allow manual override of the currently built-in automatic scaling and offsetting of the radialprofile model when used for interpolation.

12. Add an option to exclude saturated pixels when extracting sources from an image.

13. Add a tool that measures distances on the displayed image.

14. Add basic parameter settings as differentiating labels on the graphs.

15. Add a new Keep Zaps button and eponymous functionality for the pick/zap tool.

The default maximum image size is $5000 \times 5000$ pixels, which has been tested on a computer equipped with 2 Gbytes of random-access memory. The maximum-image-size setting under the Preferences menu can be increased to accommodate larger images (assuming the user's machine has sufficient memory) and, in conjunction with this increase, the user may have to start up APT with a larger heap-space setting. Currently, java -mx1024M APT is executed in APT.csh, which is the APT C-shell startup script for UNIX-based platforms, and in APT.bat, which is the batch file for starting up APT on Windows machines. Mac users running APT as a double-click application can similarly edit this specification in the Contents / Info.plist subdirectory of the APT package (before installing the package in the Mac Applications directory).

Finally, some of the Spitzer Science Center Spot/Leopard Java classes for conversion from pixel to celestial coordinates have been incorporated into APT. However, the user is cautioned that only the gnomonic (tangent), orthographic, Cartesian, and Aitoff (for FITS-viewing only) projections are implemented. Only equatorial coordinates are computed (no Galactic coordinates). Geometric image distortion, up to the ninth polynomial order, is handled by the gnomonic projection only for cases where

CTYPE1 = 'RA-TAN-SIP'

is specified in the image's FITS header; see Shupe et al. (2005) for a description of the SIP convention. In the absence of the -SIP suffix in the CTYPE1 and CTYPE2 settings for the gnomonic projection, APT will attempt to read and apply the $P V_{i-j}$ distortion keywords generated by SCAMP for the image's astrometrical calibration (Bertin 2006a), which is up to seventh polynomial order, identical to the SExtractor implementation. Other types of projections and geometric-distortion representations in the astrometric calculations may be implemented as the need arises.

\section{CONCLUSION}

In this article, we have introduced important new software to the astronomical community, called Aperture Photometry Tool or APT, which facilitates the visualization of aperture photometry in a variety of ways. APT is appropriate, useful, and convenient for professional work. It is also playing a vital role in educating the next generation of astronomers in the United States and abroad. There are indubitably many reasons that give APT a purpose in today's world, and chief among them are its graphical user interface and the critical user feedback it provides, its rich medley of tools, machine independence, ease of installation, and intuitive use. Validation of APT has been done by comparing several large statistical samples of similar results from SExtractor and APT, which is documented in a companion article appearing in this issue of the PASP (Laher et al. 2012). It is expected that APT will continue to be upgraded over time, both to remove any remaining bugs and to augment the software with new functionality and capabilities.

We thank the beta testers. In particular, Tom Jarrett, Seppo Laine, Alberto Crespo-Noriega, Bill Reach, Jeonghee Rho, and 
Nancy Silbermann made numerous helpful suggestions. Inga Saathoff conducted invaluable beta testing in Germany. We are also grateful to Xiuqin Wu, Trey Roby, Loi Ly, and Booth Hartley for generous expert Java programming help and the use of some of their Java classes. We also thank Benne W. Holwerda for his insightful suggestions and manuscript corrections. Aperture Photometry Tool's motto Inviso notitia and logo are copyright (C) 2012 by the California Institute of Technology.

\section{REFERENCES}

Bertin, E. 2006a, ASP Conf. Ser. 351, Astronomical Data Analysis Software and Systems XV (San Francisco: ASP), 112

2006b, SExtractor User's Manual, Ver. 2.5 (Paris: IAP)

$\rightarrow$ Bertin, E., \& Arnouts, S. 1996, A\&AS, 117, 393

Da Costa, G. S. 1992, ASP Conf. Ser. 23, Astronomical CCD Observing and Reduction Techniques (San Francisco: ASP), 90

Daou, D., Pompea, S., \& Thaller, M. 2004, BAAS, 36, 1500

Holwerda, B. W., 2005, Source Extractor for Dummies (Baltimore: STScI)

Howell, S. B. 1992, ASP Conf. Ser. 23, Astronomical CCD Observing and Reduction Techniques (San Francisco: ASP), 105

Joye, W. A., \& Mandel, E. 2003, ASP Conf. Ser. 295, Astronomical Data Analysis Software and Systems XII (San Francisco: ASP), 489 $\rightarrow$ Laher, R. R., Rebull, L. M., Gorjian, V., Masci, F. J., Fowler, J. W., Helou, G., \& Kulkarni, S. R., et al. 2012, PASP , 124, 764

Rebull, L. M., Gorjian, V., Squires, G., \& Team, NITARP2011, BAAS, 43, 248.11

$\rightarrow$ Rebull, L. M., Johnson, C. H., Hoette, V., Kim, J. S., Laine, S., Foster, M., Laher, R., Legassie, M., 2011, AJ, 142, 25

Shupe, D. L., Moshir, M., Li, J., Makovoz, D., Narron, R., \& Hook, R. N. 2005, in ASP Conf. Ser. 347, Astronomical Data Analysis Software and Systems XIV (San Francisco: ASP), 491

Tody, D. 1986, Proc. SPIE, 627, 733

—. 1993, in ASP Conf. Ser. 52, Astronomical Data Analysis Software and Systems II (San Francisco: ASP), 173

Wells, D. C., Greisen, E. W., \& Harten, R. H. 1981, A\&AS, 44, 363 